ARTICLE

\title{
Cell-surface localization of Pellino antagonizes Toll-mediated innate immune signalling by controlling MyD88 turnover in Drosophila
}

Shanming $\mathrm{Ji}^{1,2, \star}$, Ming Sun ${ }^{1, \star}$, Xiudeng Zheng ${ }^{3, \star},{\text { Lin } \mathrm{Li}^{1} \text {, Liwei Sun }}^{1,2}$, Dahua Chen $^{2} \&$ Qinmiao Sun ${ }^{1}$

Innate immunity mediated by Toll signalling has been extensively studied, but how Toll signalling is precisely controlled in balancing innate immune responses remains poorly understood. It was reported that the plasma membrane localization of Drosophila MyD88 is necessary for the recruitment of cytosolic adaptor Tube to the cell surface, thus contributing to Toll signalling transduction. Here we demonstrate that Drosophila Pellino functions as a negative regulator in Toll-mediated signalling. We show that Pellino accumulates at the plasma membrane upon the activation of Toll signalling in a MyD88-dependent manner. Moreover, we find that Pellino is associated with MyD88 via its CTE domain, which is necessary and sufficient to promote Pellino accumulation at the plasma membrane where it targets MyD88 for ubiquitination and degradation. Collectively, our study uncovers a mechanism by which a feedback regulatory loop involving MyD88 and Pellino controls Toll-mediated signalling, thereby maintaining homeostasis of host innate immunity.

\footnotetext{
${ }^{1}$ State Key Laboratory of Biomembrane and Membrane Biotechnology, Institute of Zoology, Chinese Academy of Sciences, Beijing 100101, China. ${ }^{2}$ State Key Laboratory of Reproductive Biology, Institute of Zoology, Chinese Academy of Sciences, Beijing 100101, China. ${ }^{3}$ Centre for Computational and Evolutionary Biology, Institute of Zoology, Chinese Academy of Sciences, 1 Beichen West Road, Chaoyang District, Beijing 100101, China. * These authors contributed equally to this work. Correspondence and requests for materials should be addressed to Q.S. (email: qinmiaosun@ioz.ac.cn) or to D.C. (email: chendh@ioz.ac.cn).
} 
nnate immunity is the first line of host defence against invading microorganisms, including bacteria, fungi and viruses ${ }^{1}$. Understanding the mechanisms of innate immune signalling regulation is critical in immunology. Drosophila has provided an ideal model to study the genetic regulation of innate immunity, given its powerful in vivo genetics ${ }^{2-4}$. In Drosophila, the Toll and the immune deficiency (IMD) pathways are involved in innate immune responses by controlling the transcriptional expression of genes encoding antimicrobial peptides, and both pathways are evolutionarily conserved in mammals ${ }^{3,4}$. While the Toll pathway is highly analogous to the interleukin-1 receptor (IL-1R) and MyD88-dependent TLR pathways in mammals, the regulation of IMD pathway is similar to the tumour necrosis factor receptor pathway ${ }^{5,6}$.

In the IMD pathway, Gram-negative bacterial infection results in the expression of genes encoding antimicrobial peptides such as Attacin, Cecropin and Diptericin ${ }^{7,8}$. Expression of these antimicrobial genes requires the signal-dependent cleavage and subsequent nuclear translocation of Relish, a member of the $N F-\kappa B$ family of transcription factors ${ }^{7,8}$. In contrast, in the Toll pathway Spaetzle is proteolytically activated by a serine protease cascade in the circulatory system and binds to the transmembrane receptor Toll upon Gram-positive bacteria or fungal infection ${ }^{9-11}$. The subsequent intracellular cascade results in the dissociation of the NF- $\mathrm{BB}$ family member Dorsal or Dif (Dorsal-related immunity factor) from its inhibitor Cactus, the Drosophila homologue of IкB-like protein, through the recruitment of the myeloid differentiation factor 88 homologue $(\mathrm{MyD} 88)^{12,13}$, and the Drosophila IL-1R-associated kinases, Pelle and Tube (Drosophila IRAK-1 and IRAK-4 homologues). Activated Dif thus accumulates in the nucleus and subsequently promotes the expression of downstream target genes in adult flies, such as drosomycin and metchnikowin ${ }^{10,14}$.

Plasma membrane localization of receptors and their associated adaptors has been proposed to be important for signalling transduction, although internalized receptors through endosomes could also promote signalling activity ${ }^{15,16}$. In the Drosophila Toll signalling pathway, besides the Toll receptor, receptor-associated adaptor MyD88 is also localized on the plasma membrane through its C-terminal domain in a phosphoinositide-bindingdependent manner ${ }^{17,18}$. It was recently reported that plasma membrane localization is necessary and sufficient for MyD88 to mediate Toll signalling activity ${ }^{17}$, emphasizing the importance of plasma membrane localization of the Toll receptor and MyD88 for signalling transduction. However, how the signalling activity through plasma membrane localization is regulated remains elusive.

Pellino was initially identified as a Pelle interacting protein in Drosophila ${ }^{19}$. In mammals, three members of the Pellino family have been characterized, including Pellino1, Pellino2 and Pellino3, which were previously shown to function as ubiquitin E3 ligases to regulate TLR-mediated innate immune responses ${ }^{20-}$ 24. In contrast, the Drosophila genome harbours only one pellino gene that encodes Drosophila Pellino protein. It was reported that the Drosophila Pellino functions as a positive regulator in the Drosophila Toll-mediated innate immune pathway ${ }^{25}$; however, the molecular basis of the action of Drosophila Pellino is unknown.

In an effort to investigate the potential role of the RINGcontaining ubiquitin E3 ligases in the innate immunity pathway, we performed a cell-based luciferase reporter screen. Surprisingly, we found that the Drosophila Pellino acts as a negative regulator in both the S2 cell culture and the in vivo system to control Drosophila Toll-mediated innate immune pathway. Pellino accumulates at the plasma membrane upon Toll pathway activation in a MyD88-dependent manner. Moreover, Pellino associates with MyD88 via its CTE domain (a phosphoinositidebinding domain at the C-terminal of MyD88) to target MyD88 for ubiquitination and degradation. Thus, our study unravels a novel feedback regulatory mechanism involving MyD88 and Pellino that contributes to robustness of Toll signalling transduction.

\section{Results}

Pellino is a potential negative regulator in Toll signalling. To understand how the ubiquitination pathway regulates Toll-mediated innate immunity signalling, we employed the Drosophila S2 cell system and performed a cell-based RNAi screen to identify novel ubiquitin E3 ligases involved in Tollmediated innate immunity. The firefly luciferase gene was placed under the control of the drosomycin promoter $^{26}$. Activation of the Toll pathway by expression of the active form of Toll receptor $^{11,27}$, Toll ${ }^{\Delta \mathrm{LRR}}$, resulted in activation of luciferase expression controlled by the drosomycin promoter (Drs-luc) in both dose- and time-dependent manners (Fig. 1a and Supplementary Fig. 1a). Consistent with the results obtained from the luciferase reporter assays, Toll ${ }^{\Delta \mathrm{LRR}}$ also increased expression levels of endogenous drosomycin and metchnikowin mRNAs (Supplementary Fig. 1b,c). The dsRNA library used in this screen contained 134 dsRNAs targeting most known and predicted genes encoding Drosophila RING-containing ubiquitin E3 ligases (RING-E3s). RING-E3s, collectively representing the large majority of E3s, have been linked to the control of numerous cellular processes, such as DNA repair, cell cycle control and host defence ${ }^{28,29}$. The library was analysed to identify candidate genes that affected the reporter expression of Drs-luc. Among the 134 specific dsRNAs designed against Drosophila RING-E3s, treatment with one dsRNA targeting CG5212, which encodes Drosophila Pellino, resulted in a significant upregulation of luciferase activity driven by the drosomycin promoter, compared with control dsRNA treatment (Supplementary Fig. 2). This suggested a potential negative role of Pellino in regulating Drosophila Toll-mediated innate immunity.

Pellino has a negative role in Toll-mediated innate immunity. Mammalian Pellino proteins, including three members (Pellino1, Pellino2 and Pellino3), regulate the TLR-mediated innate immune responses ${ }^{21-24}$. In contrast, the Drosophila genome harbours only one pellino gene that has been proposed previously to function as a positive regulator in Drosophila Toll-mediated innate immune pathway ${ }^{25}$. Because of this inconsistency, we characterized the exact role of Drosophila Pellino, and sought to understand the molecular mechanism underlying its action in the Drosophila innate immunity pathway.

We designed three pellino dsRNAs independent of the dsRNA used in the RNAi screen. As shown in a western blot assay, these dsRNAs targeting to two non-overlapping regions of the ORF and the $3^{\prime}$ UTR of the pellino gene efficiently knocked down Pellino expression in S2 cultured cells (Fig. 1b). Using the Drs-luc reporter assay, we found that knockdown of pellino significantly enhanced the Toll signalling activity stimulated by overexpression of the active form of Toll receptor compared with controls (Fig. 1b). We next examined the expression of antimicrobial peptide genes in S2 cells by performing quantitative RT-PCR (qRT-PCR) assays. In agreement with the results obtained from the luciferase reporter assays, expression levels of endogenous drosomycin, metchnikowin and defensin mRNAs also increased when pellino was knocked down in the Toll ${ }^{\Delta \mathrm{LRR}}$ overexpressing S2 cells, compared with the control (Fig. 1c-e). Consistent results were obtained when Drosomycin and Defensin proteins were measured (Fig. 1f). In addition, rescue experiments showed that 
a

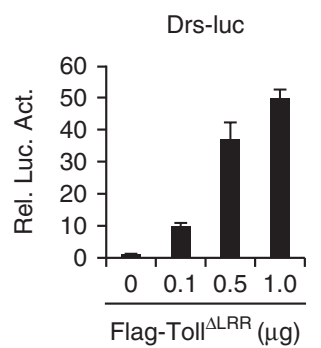

WB: Flag $\longrightarrow 45 \mathrm{kDa}$

WB: actin $=-0.43 \mathrm{kDa}$

d

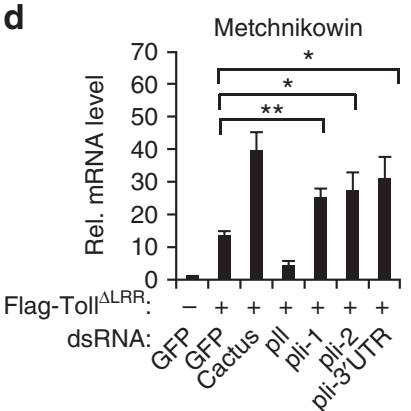

b

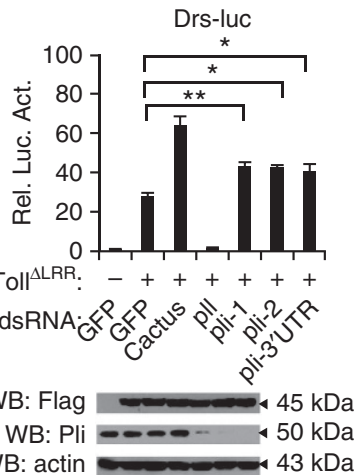

e

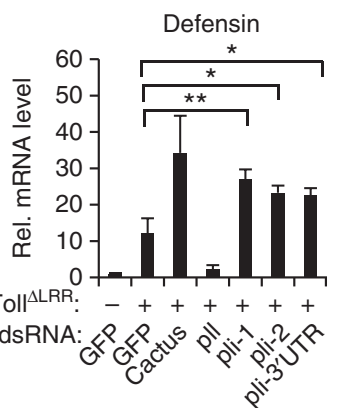

c

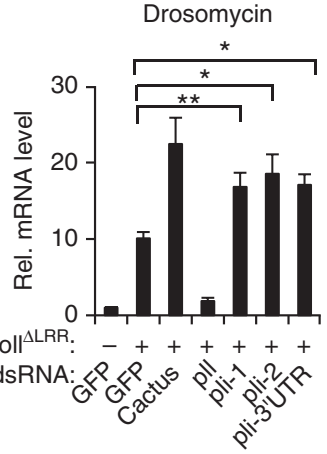

f

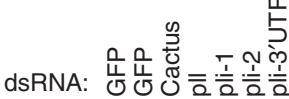

Flag-Toll ${ }^{\Delta \mathrm{LRR}:}-++++++$

WB: Drs $-18 \mathrm{kDa}$

WB: Def $->-19 \mathrm{kDa}$

WB: actin $43 \mathrm{kDa}$ g

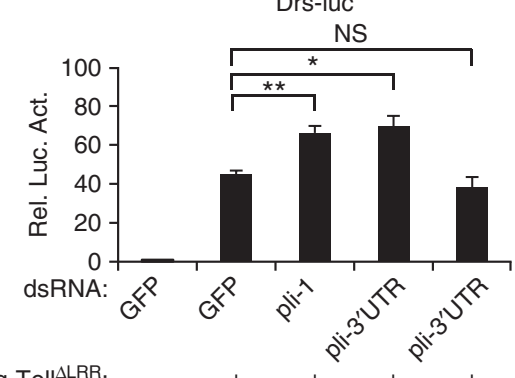

Flag-Toll $^{\Delta \mathrm{LRR}}$
Myc-Pli

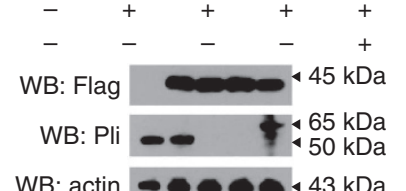

h

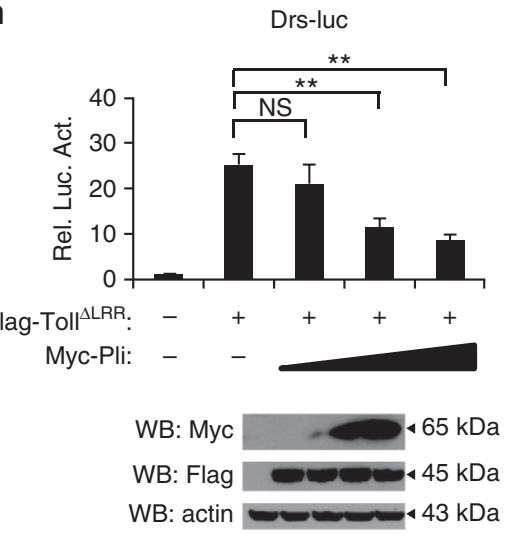

Figure 1 | Pellino negatively regulates Toll signalling in Drosophila. (a) S2 cells were transfected with the expression empty or different doses of Toll ${ }^{\Delta L R R}$ vectors together with Drs-luc and Renilla-luc plasmids. Thirty-six hours after transfection, cells were lysed for luciferase assays (upper panel) and immunoblotting assays (lower panel). Luciferase activity was measured and normalized based on renilla luciferase activity. Error bars represent s.d. ( $n=3$ ). (b) DsRNAs targeting gfp, cactus (negative control), pelle (pll, positive control) or three different regions of pellino were treated in S2 cells for $48 \mathrm{~h}$, then the empty vector or Toll ${ }^{\Delta L R R}$ plasmids were transfected in S2 RNAi cells with Drs-luc and Renilla-luc plasmids. At $36 \mathrm{~h}$ post-transfection, cells were lysed for luciferase assays (upper panel), and immunoblotting with a Pellino-specific antibody to confirm RNAi efficiency (lower panel). Error bars represent s.d. $(n=3)$. (c-e) S2 cells were treated with dsRNAs targeting gfp, cactus, pelle or pellino. After $48 \mathrm{~h}$ treatment, cells were transfected with DNA vector expressing Toll ${ }^{L R R}$. At $36 \mathrm{~h}$ post-transfection, total RNA was isolated for quantitative RT-PCR to determine transcriptional levels of drosomycin (c) metchnikowin (d) and defensin (e). Error bars represent s.d. $(n=3)$. (f) $\mathrm{S} 2$ cells were pretreated with gfp, cactus, pelle and pellino dsRNAs for $48 \mathrm{~h}$ and then were transfected with vectors expressing Toll ${ }^{\Delta L R R}$. Forty-eight hours after transfection, cells were lysed for western blot assays to measure protein levels of Drosomycin and Defensin. Actin is shown as loading control. (g) S2 cells were treated with dsRNA (as indicated) for $48 \mathrm{~h}$, reporter plasmids and the indicated expression vectors were transfected into dsRNA knockdown cells. After $36 \mathrm{~h}$, cells were lysed for luciferase assays (upper panel), and immunoblotting assays (lower panel). Error bars represent s.d. $(n=3)$. (h) S2 cells were transfected with vector or expression constructs as indicated. Thirty-six hours after transfection, cells were extracted for reporter assays (upper panel) and immunoblotting assays performed with the indicated antibodies (lower panel). Error bars represent s.d. $(n=3)$. For data from $(\mathbf{b}-\mathbf{e}, \mathbf{g}, \mathbf{h})$, the two-tailed Student's $t$-test was used to analyse statistical significance. ${ }^{\star} P<0.05,{ }^{\star \star} P<0.01, \mathrm{NS}$, no significance, versus control groups.

overexpression of Pellino appeared to reduce the increased activity of the drosomycin promoter by a pellino dsRNA that targeted the $3^{\prime}$ UTR of the pellino gene (Fig. 1g). Consistent with the above results, overexpression of Pellino inhibited luciferase activity by the drosomycin promoter induced by Toll ${ }^{\Delta \mathrm{LRR}}$ in a dose-dependent manner (Fig. 1h). These findings suggest that Pellino has a negative role in the Toll-mediated innate immune pathway in cultured cells. 
Pellino is dispensable for the IMD pathway. Given that Pellino is involved in the Drosophila Toll-mediated innate immunity pathway, we then determined whether Pellino also regulated the IMD signalling pathway, and established another reporter system, Att-luciferase, in which the luciferase was driven by the attacin gene promoter, which responds to IMD immune signalling. As shown in Fig. 2a, overexpression of the active form of PGRP-LCa (PGRP-LCa $\left.{ }^{\text {TM }+ \text { Intra }}\right)^{30,31}$ significantly induced luciferase expression in a dose-dependent manner. In contrast to the role of Pellino in regulating the Toll pathway, knockdown of pellino had no effect on luciferase activity induced by expression of PGRP-LCa ${ }^{\mathrm{TM}}+$ Intra in S2 cells (Fig. 2b). Overexpression of pellino did not affect PGRP-LCa TM+Intra induced Att-luciferase activity in transfected S2 cells (Fig. 2c). Thus, Pellino might be dispensable for attacin expression. To confirm this, we performed quantitative PCR assays to measure mRNA levels of attacin (att), cecropin A1 (cecA1) and diptericin $(d p t)$ genes, three downstream antimicrobial genes in the IMD a

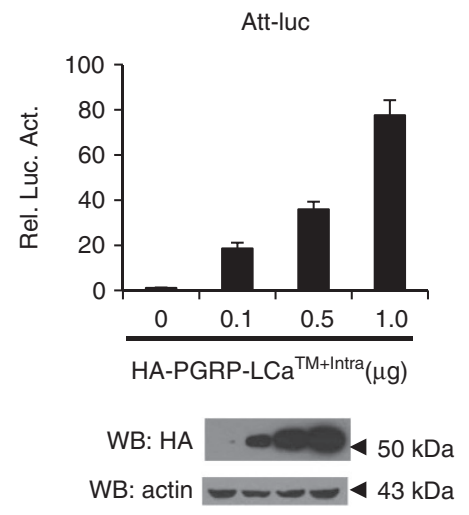

C
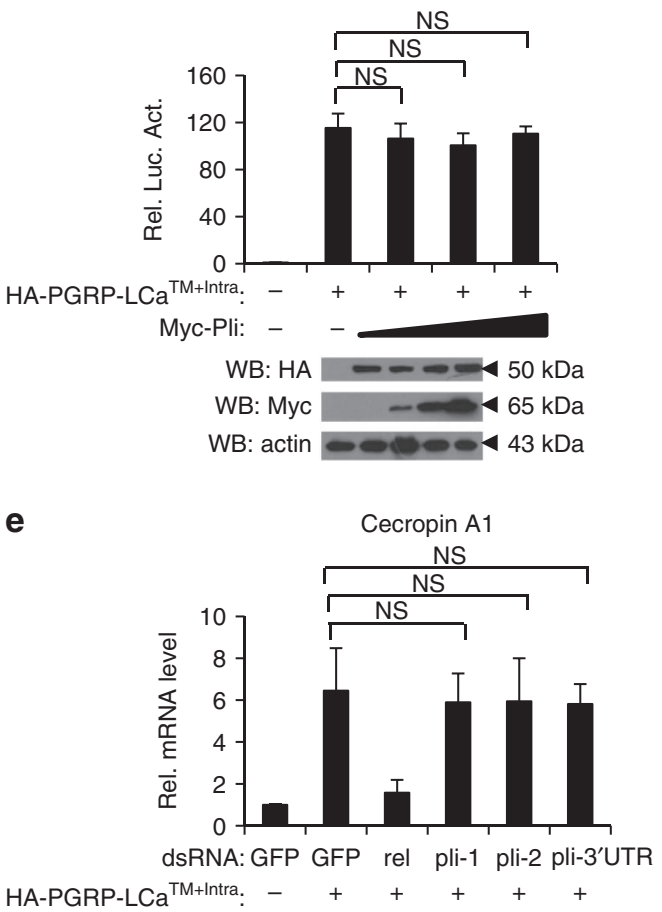

b

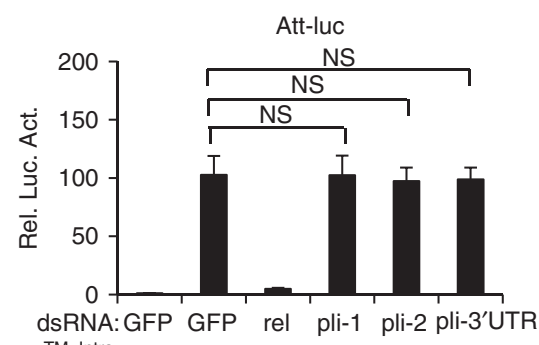

HA-PGRP- LCa ${ }^{\text {TM+Intra: }}$

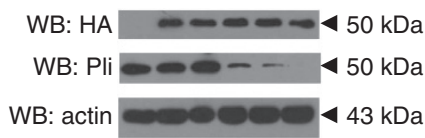

d

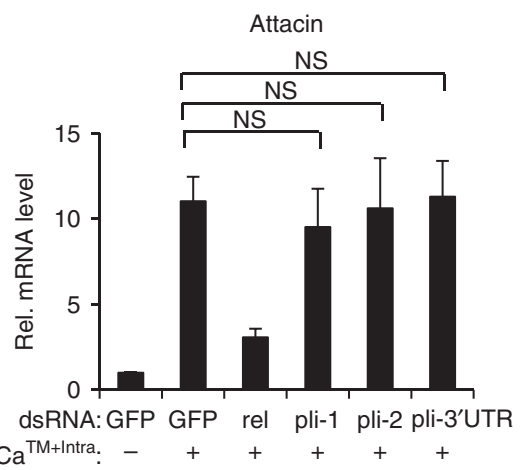

f

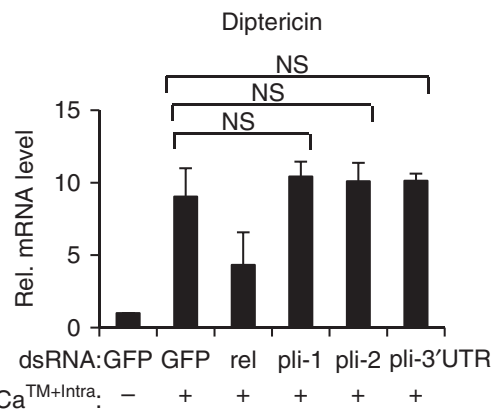

Figure 2 | Pellino is not involved in the Drosophila IMD pathway. (a) S2 cells were transiently transfected with an expression empty vector or increased doses of the PGRP-LCaTM + Intra (PGRP-LCa active form) vectors together with a luciferase reporter construct driven by the promoter of attacin gene (Att-luc). Renilla luciferase plasmid was used as an internal control. Thirty-six hours after transfection, luciferase activity was measured and normalized based on renilla luciferase activity (upper panel), and immunoblotting assays were performed (lower panel). Error bars represent s.d. ( $n=3$ ). (b) DsRNAs targeting gfp, relish (rel, positive control) or three different regions of pellino were used to treat S2 cells for $48 \mathrm{~h}$, then empty vector or HA-PGRP$\mathrm{LCa}^{\mathrm{TM}}+$ Intra plasmids were transfected in dsRNA-treated S2 cells with Att-luc and Renilla-luc plasmids. At $36 \mathrm{~h}$ post-transfection cells were lysed for luciferase assays (upper panel) and immunoblotting performed to confirm RNAi efficiency (lower panel). Error bars represent s.d. ( $n=3$ ). (c) DNA vector expressing PGRP-LCaTM + Intra and increasing amounts of expression Myc-Pellino constructs were transfected into S2 cells with Att-luc and Renilla-luc plasmids. At $36 \mathrm{~h}$ post-transfection, cells were lysed for luciferase assay (upper panel), and immunoblotting to check protein expression levels (lower panel). Error bars represent s.d. $(n=3)$. (d-f) S2 cells were treated with dsRNAs targeting pellino, relish or gfp. After $48 \mathrm{~h}$ treatment, cells were transfected with DNA vector expressing PGRP-LCa ${ }^{\mathrm{TM}}+$ Intra. At $36 \mathrm{~h}$ post transfection, total RNA was isolated for quantitative RT-PCR to check transcriptional levels of attacin $(\mathbf{d})$, cecropin $A 1(\mathbf{e})$ and diptericin $(\mathbf{f})$. Error bars represent s.d. $(n=3)$. For data from $\mathbf{b}$-f, the two-tailed Student's $t$-test was used to analyse statistical significance. NS, no significance, versus control groups. 
pathway, in pellino knockdown and control S2 cells. We found that knockdown of pellino did not affect the induction of att, cecA1 or $d p t$ expression (Fig. 2d-f). A recent study has suggested that ecdysone could potentially induce expression of target genes of the IMD pathway by triggering PGRP-LC expression ${ }^{32}$. We next sought to test whether Pellino regulates the IMD signalling induced by ecdysone, and found that neither overexpression of Pellino nor knockdown of Pellino has apparent effect on the IMD signalling activity induced by the ecdysone treatment (Supplementary Fig. 3a-e). Collectively, our findings suggest that Pellino functions specifically in the Drosophila Tollmediated, but not in the IMD-mediated innate immune pathway.

Pellino regulates Toll-mediated immune responses in vivo. Next, we determined whether Pellino controlled the Toll-mediated immune pathway in vivo. As previously described ${ }^{33}$, we generated several transgenic lines, $\mathrm{P}\{U A S p$-artmiR-pellino $\}$, in which artificial miRNAs that specifically target the pellino gene were under the control of the UASp promoter. As shown in Fig. 3a and Supplementary Fig. 4a, ubiquitous expression of artmiR-pellino by the Tub-gal4 driver efficiently downregulated Pellino expression, but caused animal lethality at the late larval stage, which was consistent with previous findings that pellino is an essential gene for animal development ${ }^{25}$. To assess the biological role of Pellino in regulating innate immunity in the adult stage of Drosophila, we first employed the ubiquitous Gal4-Gal80ts driver system and took advantage of the temperature-dependent activity of the Gal4 that is negatively regulated by the Gal80 to control expression of artmiR-pellino specifically at the adult stage. By using this system, crosses were performed and the resulting progeny were initially raised at the permissive temperature $\left(18^{\circ} \mathrm{C}\right)$ for the Gal80ts inhibitor to repress the Gal4 activity. Once adult eclosion, the newly progeny were then shifted to $29^{\circ} \mathrm{C}$ to allow artmiR-pellino expression. The 6-day-old control and pellino knockdown flies were then infected by Gram-positive bacterium Micrococus luteus (M. luteus), a widely used bacterial strain that induces activation of the Tollmediated immune response and expression of AMPs. As shown in Supplementary Fig. 4b,c, expression levels of drosomycin and metchnikowin mRNAs were higher in pellino knockdown flies than that in controls. Since the fat body is the Drosophila immune organ, we then employed the fat body-specific driver, $\mathrm{Cg}$-gal4, to knock down pellino expression in fat bodies, and obtained consistent results (Fig. 3b-e). To further confirm these observations, we next used other bacterial and fungal pathogens, such as Enterococcus faecalis (E. faecalis) and Aspergillus fumigatus (A. fumigatus), to infect wild-type and pellino knockdown flies. As shown in Supplementary Fig. $4 \mathrm{~d}-\mathrm{g}$, knockdown of pellino indeed enhanced Toll signalling activity induced by these pathogens. To test whether overexpression of pellino downregulates Toll signalling, we infected flies overexpressing pellino by the Cg-gal4 driver with M. luteus, E. faecalis or A. fumigatus and found that elevation of Pellino expression in fat bodies significantly reduced the expression levels of drosomycin and metchnikowin, when compared with controls (Fig. 3f,g and Supplementary Fig. $4 \mathrm{~d}-\mathrm{g}$ ). In agreement with the data from qRT-PCR assays, consistent results were obtained, when protein levels of Drosomycin and Defensin were measured (Supplementary Fig. 4h). Collectively, our results strongly argue that Pellino functions as a negative regulator to control Tollmediated innate immunity in the Drosophila immune organ, the fat bodies.

Pellino regulates invading pathogen growth. Given that Pellino has a negative role in controlling the Toll-mediated innate immunity pathway, we tested the biological impact of Pellino, and examined whether pellino influenced $M$. luteus growth in host flies by performing bacterial load assays as previously described ${ }^{9}$. Bacterial colony-forming unit (CFU) assays demonstrated the quantity of M. luteus colonies from pellino knockdown flies was lower than that from control flies (Fig. 3h), suggesting Pellino might influence the breeding of $M$. luteus. Comparison of the survival rate of control and pellino knockdown flies infected with M. luteus showed that pellino knockdown flies had a higher survival rate than controls (Fig. 3j). To confirm these observations, we tested whether fat body-specific overexpression of Pellino influenced the breeding of $M$. luteus. The quantity of $M$. luteus colonies from flies with fat body-specific overexpression of pellino was increased (Fig. 3i) compared with controls. In addition, the pellino overexpressed flies had a lower survival rate than controls (Fig. 3k). Thus, Pellino may influence the host defence ability against Gram-positive bacterial infection.

Pellino physically interacts with MyD88. To understand the molecular mechanism underlying the action of Pellino in the Toll-mediated innate immune pathway, we examined whether Pellino interacted with known components of the Toll pathway. Pellino has been previously reported to interact with Pelle in an yeast two-hybrid analysis ${ }^{1}$. Consistent with this, our co-immunoprecipitation assays showed that Pellino associated with Pelle in transfected S2 cells (Fig. 4a and Supplementary Fig. 5a). We then tested whether Pellino also associated with Tube and MyD88, other key components of the Toll signalling pathway ${ }^{13}$. Co-immunoprecipitation assays demonstrated that Tube and MyD88 were also associated with Pellino (Fig. 4b and Supplementary Fig. 5b,c). Previous studies showed that Tube and Pelle formed a functional complex with MyD88 to regulate Toll signalling. We therefore sought to test whether Pelle or Tube affects the MyD88-Pellino complex assembly. As shown in co-immunoprecipitation experiments, knockdown of tube or pelle (Supplementary Fig. 6) did not affect MyD88-Pellino interactions (Fig. 4c). Thus, Pellino forms a complex with MyD88 in a Pelle/ Tube independent manner.

Pellino regulates MyD88 ubiquitination and turnover. Given that Pellino functions as a ubiquitin E3 ligase and is physically associated with Pelle, Tube and MyD88, we tested whether Pellino controlled ubiquitination of these proteins by performing in vivo ubiquitination assays in transfected S2 cells. As shown in Fig. 4d, overexpression of Pellino markedly increased ubiquitination of MyD88, but did not ubiquitinate Pelle or Tube. Conversely, the conjugation of ubiquitin to MyD88 was markedly reduced when S2 cells were treated with dsRNAs of pellino (Fig. 4e), suggesting Pellino has a specific role in regulating ubiquitination of MyD88, rather than Pelle or Tube. It has been proposed that K48-linked ubiquitination is exclusively related to protein degradation, whereas K-63-linked ubiquitination likely promotes protein-protein association and signalling activation ${ }^{34}$. To determine whether Pellino-mediated MyD88 ubiquitination is linked via $\mathrm{K} 63$ or K48, we also employed a series of HA-tagged mutants of ubiquitin to perform ubiquitination assays. Overexpression or knockdown of Pellino markedly increased or reduced the ubiquitination of MyD88, respectively, when a HA-Ub-K48 mutant (all lysines in ubiquitin are mutated to arginine except K48) was used (Fig. 4f,g). In contrast, alteration of Pellino expression did not change the ubiquitination pattern of MyD88, when a HA-Ub-K63 mutant (all lysines in ubiquitin are mutated to arginine except K63) was used (Supplementary Fig. 7a,b). Thus, Pellino-mediated MyD88 ubiquitination occurs through K-48 ubiquitin linkage. In support of this, overexpression 
a

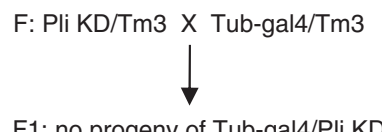

b

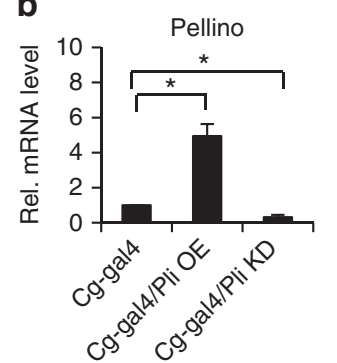

C

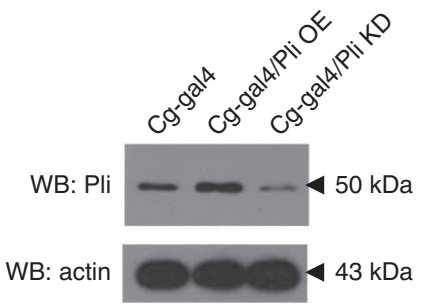

d
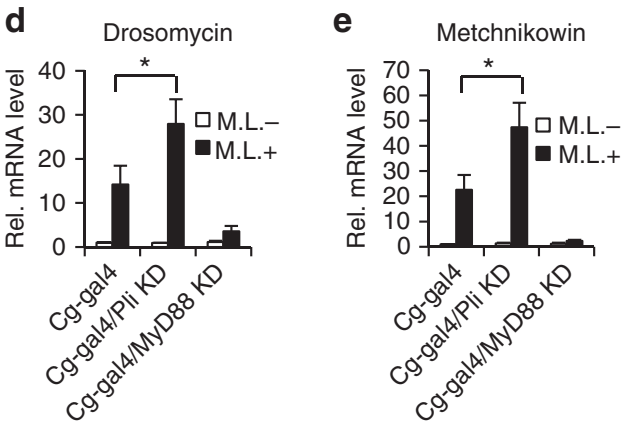

h
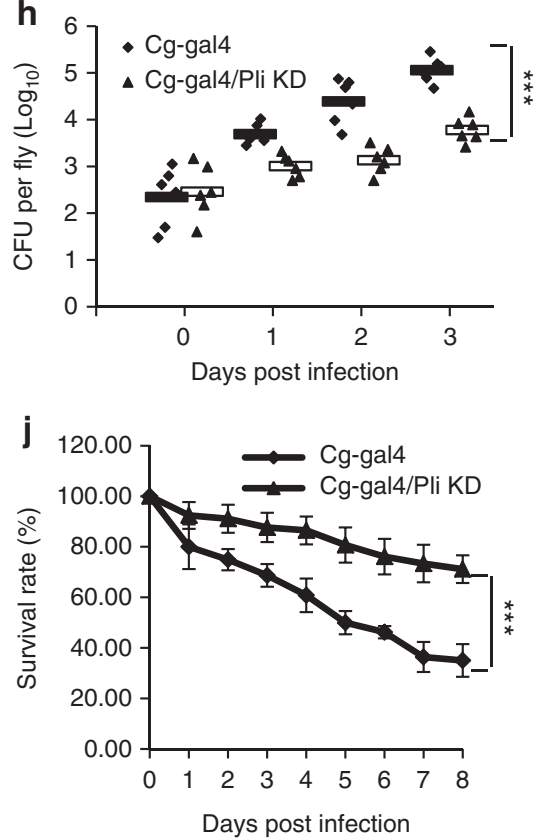

f
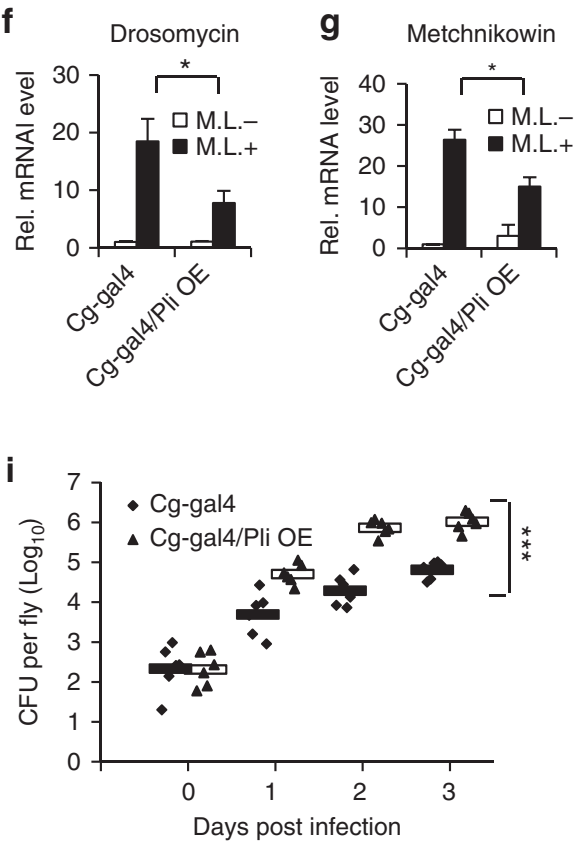

$\mathbf{k}$

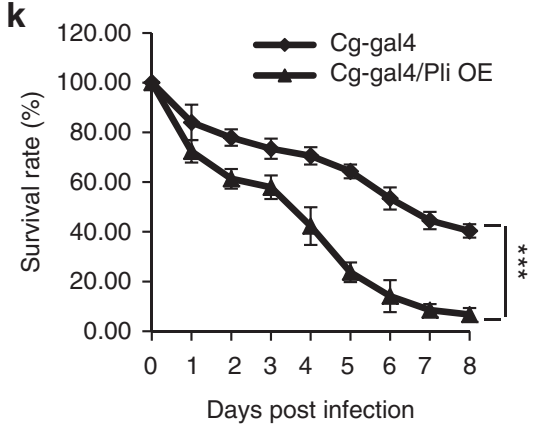

Figure 3 | Pellino regulates Toll-mediated immune responses in vivo. (a) The UAS-GAL4 strategy was used to test the in vivo function of pellino. Ubiquitous knockdown of pellino by the Tub-gal4 resulted in animal lethality at the late larval stage. (b,c) Flies containing overexpression or knockdown of pellino specifically in fat bodies by the $\mathrm{Cg}$-gal 4 driver were lysed to measure expression levels of pellino mRNA (b) or protein (c). Error bars represent s.d. $(n=3)$. (d,e) Pellino knockdown, MyD88 knockdown (positive control) and Cg-gal 4 flies (wild-type control) were infected with $M$. luteus for $12 \mathrm{~h}$ and lysed for quantitative RT-PCR analysis to measure mRNA levels of drosomycin $(\mathbf{d})$ and metchnikowin (e). Error bars represent s.d. $(n=3)$. ( $(\mathbf{f}, \mathbf{g})$ Pellino overexpression flies and controls were infected with $M$. luteus for $12 \mathrm{~h}$ and then lysed for quantitative RT-PCR analysis to measure mRNA levels of drosomycin (f) and metchnikowin $(\mathbf{g})$. Error bars represent s.d. $(n=3)$. (h,i) Pellino knockdown $(\mathbf{h})$ or overexpression (i) flies and controls were pricked with a needle previously dipped into $M$. luteus. Bacterial replication was monitored at different times as indicated. (j,k) Pellino knockdown (j) or overexpression (k) flies and controls were infected with M. luteus and monitored daily for mortality. Error bars represent s.d. $(n=3)$. For data from $\mathbf{b}$ and $\mathbf{d}-\mathbf{g}$, the two-tailed Student's $t$-test was used to analyse statistical significance. For data from $\mathbf{h}$ and $\mathbf{i}$, the rANOVA test was used to analyse the variance of repeat measures. For data from $\mathbf{j}$ and $\mathbf{k}$, the Log-rank test was used to analyse the variance of the survival rates between two groups. ${ }^{\star} P<0.05,{ }^{\star \star \star} P<0.001$, versus control groups.

of Pellino reduced the expression levels of MyD88 (Fig. 4h), whereas knockdown of Pellino increased the stability of the Myctagged MyD88 and endogenous MyD88 as well (Fig. 4i and Supplementary Fig. 7c). Additionally, we also observed an apparent reduction of endogenous MyD88 upon high levels of Toll activation (Supplementary Fig. 7d). Collectively, our results strongly argue that Pellino interacts with MyD88 and specifically regulates MyD88 ubiquitination and turnover. 
a

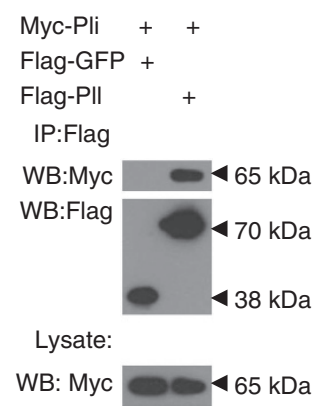

d

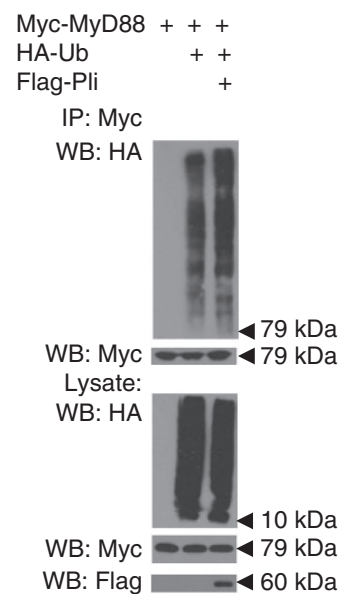

b

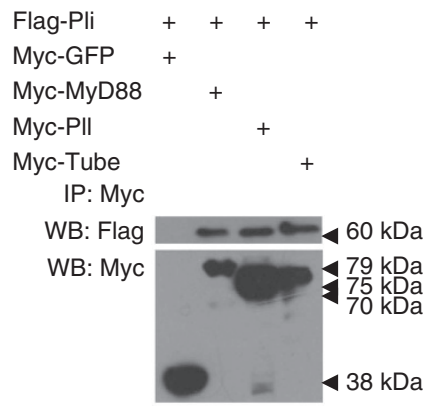

Lysate:

WB: Flag

e

Myc-MyD88 + + +

$\begin{array}{lr}\text { HA-Ub } & ++ \\ \text { pli dsRNA } & +\end{array}$

IP: Myc

WB: HA

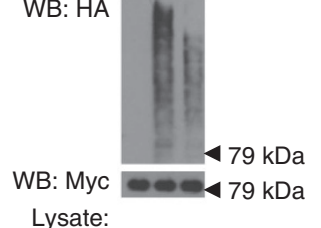

WB: HA

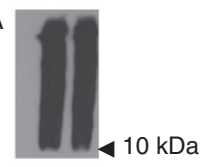

WB: Myc $=-179 \mathrm{kDa}$

WB: Pli $=450 \mathrm{kDa}$

f
C

\begin{tabular}{|c|c|c|c|}
\hline Myc-Pli & + & + & + \\
\hline Flag-MyD88 & & + & + \\
\hline pll dsRNA & & & + \\
\hline tube dsRNA & & & \\
\hline IP: Flag & & & \\
\hline
\end{tabular}

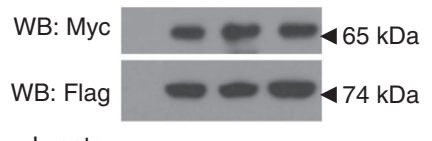

Lysate:

WB: Myc 465 kDa

g

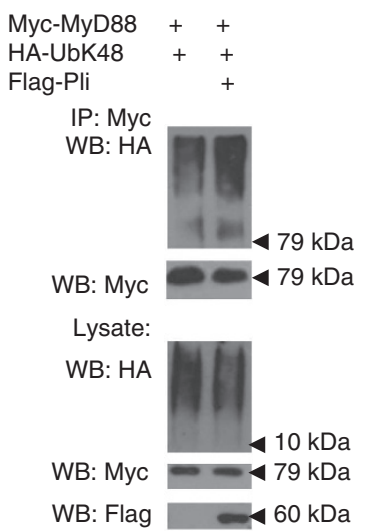

HA-UbK48 + +

pli dsRNA +

IP: Myc

WB: HA

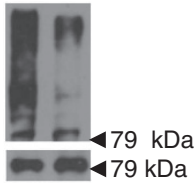

WB: Myc 579 kDa

Lysate:

WB: HA

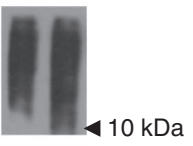

WB: Myc $=479$ kDa

WB: Pli $450 \mathrm{kDa}$ h
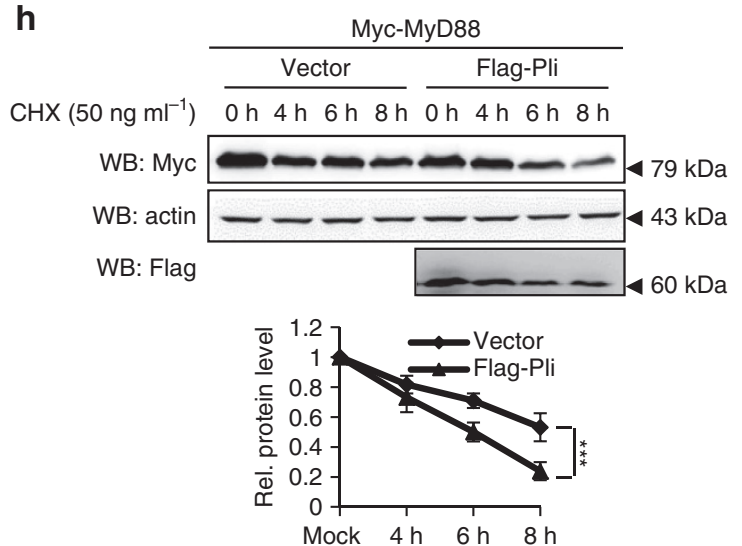

i

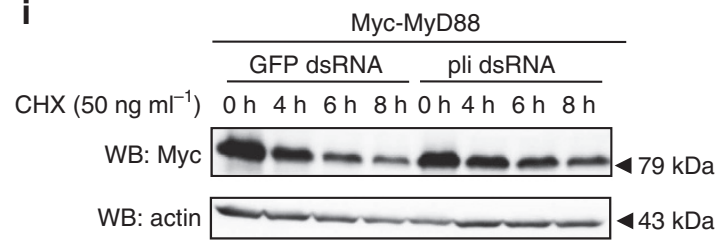

Figure 4 | Pellino regulates MyD88 ubiquitination and turnover through K-48 linkage. (a,b) S2 cells were transfected with combinations of expression plasmids as indicated. Forty-eight hours after transfection, cell lysates were prepared, immunoprecipitated with anti-Flag beads (a) or anti-Myc beads (b), followed by immunoblot analysis with the indicated antibodies. Expression levels of transfected proteins in whole-cell lysates are shown in bottom panel. (c) S2 cells were treated with the dsRNAs as indicated for $48 \mathrm{~h}$, and then transfected with combinations of expression plasmids as indicated. Forty-eight hours after transfection, cell lysates were prepared, immunoprecipitated with anti-Flag beads, followed by immunoblot analysis with the indicated antibodies. Expression levels of transfected proteins in whole-cell lysates are shown in bottom. (d,e) S2 cells were transfected with combinations of expression plasmids as indicated (d) or cells were also pretreated with pellino dsRNA (e). Cell lysates were then used to perform immunoprecipitation experiments with anti-Myc beads, followed by immunoblot analysis with anti-HA antibodies to show ubiquitination pattern of MyD88. (f,g) Similar to d and $\mathbf{e}$, except the DNA vector expressing HA-Ub-K48 was used to examine the MyD88 K48-linked ubiquitination pattern under overexpression (f) or knockdown ( $(\mathbf{g})$ of pellino condition. (h) S2 cells were transfected with expression constructs as indicated, and after $48 \mathrm{~h}$ post transfection, cells were treated with $\mathrm{CHX}\left(50 \mathrm{ng} \mathrm{ml}^{-1}\right)$ for different times, followed by immunoblotting to check MyD88 expression levels. Densitometry analysis to quantify MyD88 expression is shown in the bottom panel. Error bars represent s.d. $(n=3)$. (i) S2 cells were treated with dsRNAs targeting gfp or pellino for $48 \mathrm{~h}$, then transfected with Myc-MyD88. After $48 \mathrm{~h}$, cells were treated with $\mathrm{CHX}\left(50 \mathrm{ng} \mathrm{ml}{ }^{-1}\right)$ for different times, followed by immunoblotting to check MyD88 expression levels. Densitometry analysis to quantify MyD88 expression is shown in the bottom panel. Error bars represent s.d. ( $n=3$ ). For data from $\mathbf{h}$ and $\mathbf{i}$, the Log-rank test was used to analyse the variance of the protein stability between two groups. ${ }^{\star \star \star} P<0.001$, versus control groups. 
Toll signalling promotes accumulation of Pellino at the plasma membrane. It has been shown that Drosophila MyD88 is localized at the plasma membrane in Drosophila embryos ${ }^{18}$. Recently, an elegant study demonstrated the plasma membrane localization of MyD88 is necessary and sufficient for the signalling transduction of Toll-mediated innate immune responses ${ }^{17}$. As Pellino negatively regulates Toll signalling by controlling the ubiquitination and degradation of MyD88, Pellino might function at the plasma membrane to target MyD88. To test this, we used S2 cells to perform cell-based immunostaining assays. Pellino was expressed predominately in the cytosolic region, but small portion of Pellino staining was observed at the plasma membrane when S2 cells expressed Myc-tagged Pellino alone (Fig. 5a,b). However, Pellino predominately accumulated at
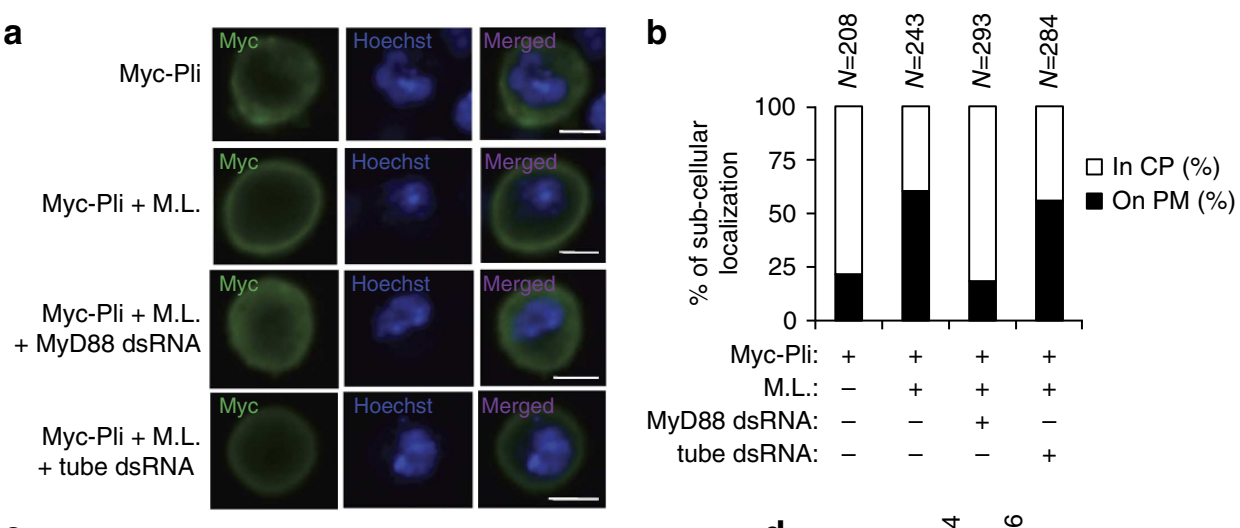

C
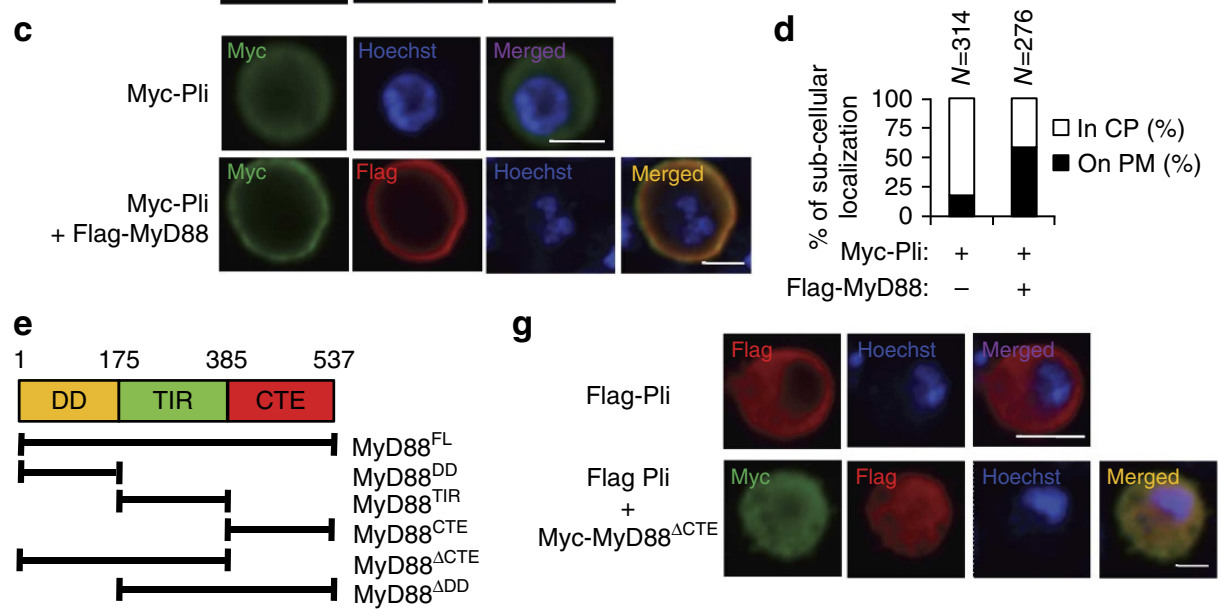

f
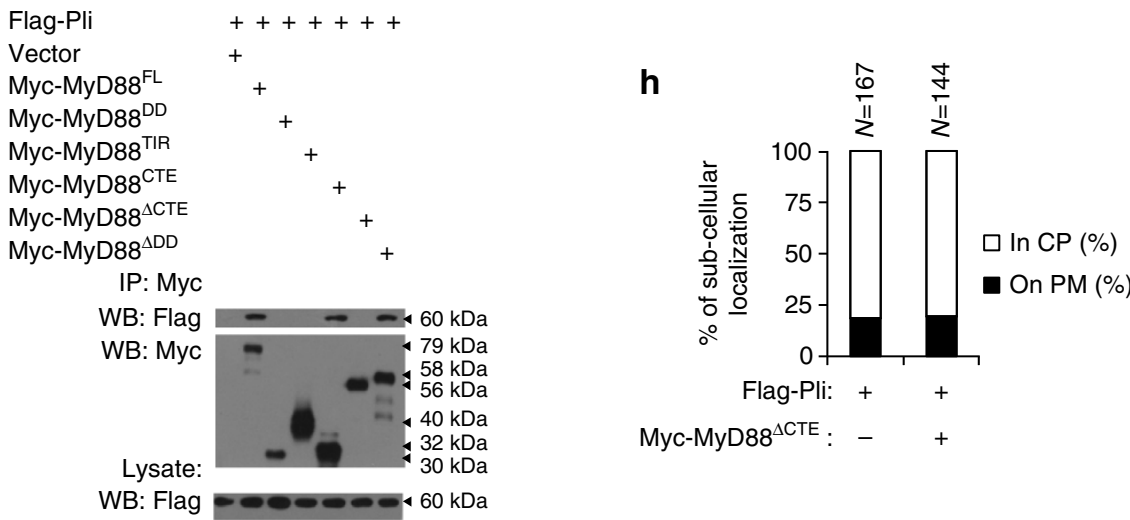

Figure 5 | Toll signalling promotes Pellino to accumulate at the plasma membrane in a MyD88-dependent manner. (a) S2 cells were transfected with Myc-tagged Pellino or also pretreated with MyD88 or Tube dsRNA, and then treated with or without pre-boiled M. luteus (ML) for $12 \mathrm{~h}$ as indicated. Cells were further stained with anti-Myc antibody and Hoechst, and imaged by confocal microscopy. Scale bars, $10 \mu \mathrm{m}$. (b) Statistical assays of plasma membrane (PM) or cytoplasmic (CP) localization of Pellino in cell samples from (a). (c,d) S2 cells transfected with Myc-Pellino alone or Myc-Pellino in combination with Flag-MyD88 were stained with Hoechst and anti-Myc antibody alone or with anti-Flag antibody as indicated and imaged by confocal microscopy(c). Scale bars, $10 \mu \mathrm{m}$. (d) Statistical assays of PM or CP localization of Pellino in cell samples from (c). (e) Schematic diagram of MyD88 and its truncated mutants. (f) S2 cells were transfected with Flag-tagged Pellino and Myc-tagged MyD88 or its truncated mutants. Forty-eight hours after transfection, cell lysates were prepared, immunoprecipitated with anti-Myc beads, followed by immunoblot analysis with the indicated antibodies. $(\mathbf{g}, \mathbf{h})$ S2 cells transfected with Flag-Pellino alone or in combination with Myc-MyD88 ${ }^{\Delta C T E}$ and stained with Hoechst and anti-Flag antibody alone or with anti-Myc antibody as indicated and imaged by confocal microscopy (g). Scale bars, $10 \mu \mathrm{m}$. (h) Statistical assays of PM or CP localization of Pellino in cell samples from $(\mathbf{g})$. 
the plasma membrane, when cells were stimulated with pretreated $M$. luteus (seen in Methods) or activated by a gain-of-function mutant Toll ${ }^{10 \mathrm{~B}},{ }^{35,36}$ (Fig. 5a,b and Supplementary Fig. 8a,b), suggesting that activation of Toll signalling is sufficient to promote the plasma membrane accumulation of Pellino. To test whether MyD88 or Tube is required for Pellino plasma membrane accumulation, we performed $M y D 88$ or Tube knockdown experiments in S2 cells, inactivation of MyD88 but not Tube markedly reduced $M$. luteus- or Toll ${ }^{10 \mathrm{~B}}$-induced Pellino plasma membrane accumulation in S2 cells (Fig. 5a,b and Supplementary Fig. 8a,b). Thus, the activation of Toll signalling promoted the accumulation of Pellino at the plasma membrane in a MyD88-dependent manner.

MyD88 CTE domain is key for MyD88-Pellino regulatory loop. We next tested whether MyD88 was sufficient to induce the plasma membrane accumulation of Pellino. Plasma membrane accumulation of Pellino significantly increased when S2 cells were co-expressed with Myc-Pellino and Flag-MyD88, compared with Pellino expressed alone in S2 cells (Fig. $5 c$,d). To investigate the molecular basis of how MyD88 promotes plasma membrane accumulation of Pellino, we identified the specific domain in MyD88 required for Pellino interactions and generated a series of mutant forms of MyD88 constructs (Fig. 5e). Co-immunoprecipitation demonstrated that although DD and TIR domains were not required for MyD88 interactions with Pellino, the MyD88 CTE domain (a phosphoinositide-binding domain at the C-terminal of MyD88) ${ }^{17}$ was essential since the mutant form of MyD88 ${ }^{\Delta \mathrm{CTE}}$ lacking CTE domain did not associate with Pellino (Fig. 5f). To test whether the CTE domain was required for MyD88 to promote the plasma membrane accumulation of Pellino, we co-overexpressed the mutant form of MyD88 ${ }^{\Delta \mathrm{CTE}}$ and Pellino in S2, and found that, unlike wild-type MyD88, MyD88 ${ }^{\Delta \mathrm{CTE}}$ failed to promote Pellino plasma membrane accumulation (Fig. $5 c, d, g$ and h). Thus, the CTE domain is required for MyD88 plasma membrane localization, MyD88-Pellino interaction and MyD88 recruitment of Pellino plasma membrane accumulation. We next tested whether the MyD88 CTE domain was involved in Pellino-mediated MyD88 ubiquitination and degradation. Either overexpression or knockdown of Pellino did not affect the ubiquitination status and stability of MyD88 ${ }^{\Delta \mathrm{CTE}}$, suggesting that Pellino regulates MyD88 ubiquitination through the CTE domain (Fig. 6a,b and Supplementary Fig. 7e). Given that the specific interaction of Pellino with the MyD88 CTE domain, we determined whether the MyD88 CTE domain was sufficient to promote plasma membrane localization by co-expression of Pellino and the MyD88 CTE domain in S2 cell cultures. Compared with the expression of Pellino alone, co-expression of Pellino with the MyD88 CTE domain resulted in significant cell-surface accumulation of Pellino (Fig. 6c,b). To determine the specificity of the CTE domain of MyD88 for the sub-cellular localization of Pellino, we tested whether MyD88 CTE was sufficient to recruit Tube onto the cell surface. Overexpression of full-length MyD88 resulted in the cellsurface accumulation of Tube, but MyD88 CTE alone did not (Fig. 6e,f). Taken together, our findings establish a model by which the activation of MyD88 promotes Pellino to accumulate onto the plasma membrane, where Pellino targets MyD88 for ubiquitination and degradation. This reciprocal regulation between MyD88 and Pellino appears to be mediated by the CTE domain of MyD88.

Role of plasma membrane localization of MyD88 and Pellino. Given that the CTE domain is essential for MyD88 plasma membrane localization and its function in Toll-mediated immune signalling transduction, we tested whether plasma membrane localization was sufficient for MyD88 function. We generated a plasma membrane-localized mutant form of MyD88, in which the SRC domain replaced the CTE domain (referred as MyD88 $\left.8^{\mathrm{CTE}-S R C}\right)^{37}$. We noted that, although a portion of protein exhibited particulate distribution, majority of MyD88 CTE-SRC localized to plasma membrane (Fig. 7b,d).We then performed a luciferase reporter assay, as shown in Fig. 7a, while expression of MyD88 ${ }^{\Delta C T E}$ failed to stimulate luciferase reporter expression, the replaced mutant form, MyD88 ${ }^{\mathrm{CTE}-\mathrm{SRC}}$, was efficient to induce Toll signalling, mimicking wild-type MyD88. This suggested that plasma membrane localization is essential for the MyD88 induction of Toll signalling. In support of this, expression of the SRC-replaced form of MyD88 CTE-SRC was sufficient to recruit the Tube protein onto the plasma membrane, given the TIR domain of Tube was required for interactions between Tube and MyD88 (Fig. 7b,c). However, the expression of the SRC-replaced form of MyD88 ${ }^{\mathrm{CTE}-S R C}$ was not sufficient to recruit Pellino to accumulate at the plasma membrane (Fig. $7 \mathrm{~d}, \mathrm{e})$, emphasizing the CTE domain was specifically required for MyD88-induced Pellino plasma membrane localization. We next tested whether overexpression of wild-type Pellino affected MyD88CTE-SRC induced Toll signalling. Overexpression of wild-type Pellino had no effect on Toll signalling induced by MyD88CTE-SRC (Fig. 7f). As the expression of membrane-localized Pellino, SRC-Pellino, enhanced its inhibitory activity on Toll signalling induced by the wild-type form of MyD88 (Fig. 7g), we then tested whether SRCPellino efficiently inhibited the function of MyD88 ${ }^{\mathrm{CTE}-S R C}$. SRCPellino inhibited Toll signalling activity induced by MyD88 $8^{\mathrm{CTE}}-$ SRC in a dose-dependent manner (Fig. 7h). Thus, our findings strongly argue that the plasma membrane localization of MyD88 and Pellino balances Toll-mediated immune signalling.

\section{Discussion}

In Drosophila, the Toll signalling is essential for embryonic development and innate immune responses ${ }^{3,4}$. However, how Toll signalling is regulated to maintain homeostasis of host innate immunity remains poorly understood. In this study, we demonstrated that Drosophila Pellino, a RING-containing E3 ligase, functions as a negative regulator in the Toll-mediated innate immunity pathway by targeting MyD88. Importantly, we observed that MyD88 was associated with Pellino and promoted Pellino accumulation onto the plasma membrane, where Pellino targeted MyD88 for ubiquitination and degradation. We thus uncovered a novel feedback regulatory loop involving MyD88 and Pellino that balanced Toll-mediated signalling, thereby maintaining homeostasis of host innate immunity.

Ubiquitin-mediated protein modification and/or degradation has a variety of roles in the regulation of many cellular and developmental processes ${ }^{38}$. The enzymatic reaction of protein ubiquitination is a coordinated three-step process involving three classes of enzymes, E1 (ubiquitin-activating enzyme), E2 (ubiquitin-conjugating enzyme) and E3 (ubiquitin ligase) ${ }^{38}$. Upon pathway activation, the MyD88-Tube-Pelle complex promotes the phosphorylation and degradation of Cactus, the Drosophila I $\mathrm{B}$ B factor, by Slimb/ $\beta \operatorname{TrCP}$ by a ubiquitin-dependent mechanism ${ }^{18,39}$. In addition, biochemical studies have revealed a mechanism through which the K-63 linkage of ubiquitin promotes the TRAF6/dTRAF2-mediated activation of IKK and $\mathrm{NF}-\mathrm{KB}^{34,40}$. These findings highlight that ubiquitination plays important roles in the regulation of Toll signalling. Members of the Pellino family are RING-containing proteins and were previously shown to function as ubiquitin E3 ligases to regulate TLR-mediated innate immune responses either positively or negatively in mammals ${ }^{20,21}$. Although the Drosophila genome has only one member, a previous study proposed that Drosophila Pellino acted as a positive regulator in Toll signalling, since 
a

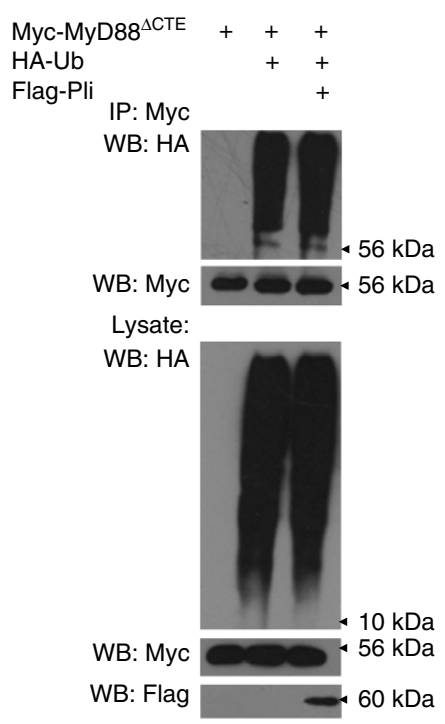

c

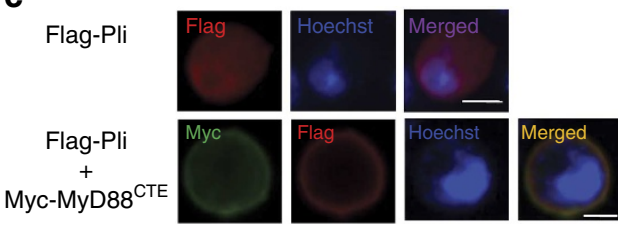

e

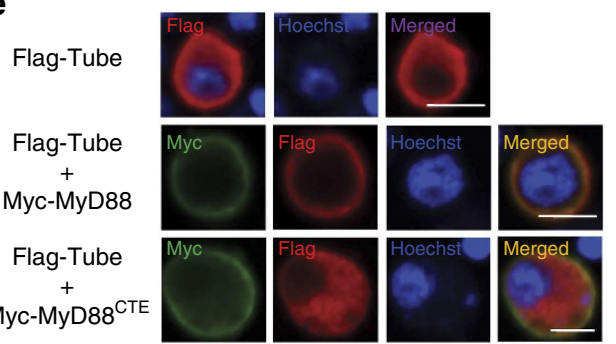

b Myc-MyD88 $8^{\triangle \mathrm{CTE}}+++$

HA-Ub ++

pli dsRNA

IP: Myc

WB: HA

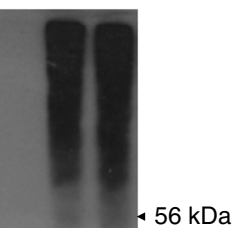

WB: Myc $56 \mathrm{kDa}$
Lysate:

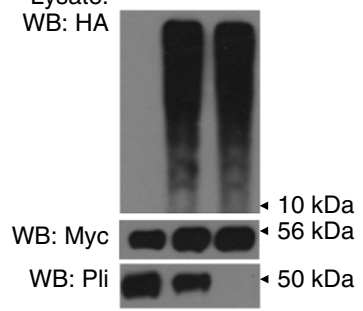

d

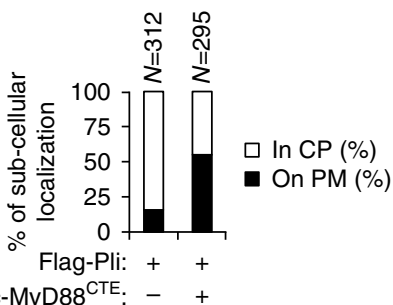

f

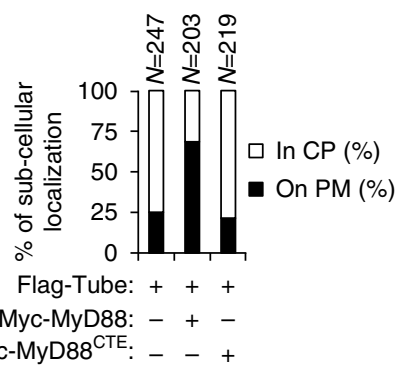

Figure 6 | Pellino-mediated MyD88 ubiquitination is dependent on CTE domain. (a,b) S2 cells were transfected with Myc-MyD88 ${ }^{\Delta C T E}$ and HA-Ub with Flag-Pellino (a), or also pretreated with pellino dsRNA (b). Cell lysates were used to perform immunoprecipitation experiments with anti-Myc beads followed by immunoblot analysis with anti-HA antibodies to show ubiquitination patterns of MyD88 ${ }^{\Delta C T E}$. Expression levels of transfected proteins and Pellino knockdown efficiency in whole-cell lysates are shown in the bottom panel. (c,d) S2 cells transfected with Flag-Pellino alone or in combination with Myc-tagged MyD88 CTE domain were stained with Hoechst and anti-Flag antibody alone or with anti-Myc antibody as indicated and imaged by confocal microscopy (c). Scale bars, $10 \mu \mathrm{m}$. (d) Statistical assays of PM or CP localization of Pellino in transfected cells in panel (c). (e,f) S2 cells transfected with Flag-Tube alone or in combination with Myc-tagged MyD88 or MyD88 CTE domain were stained with Hoechst and anti-Flag antibody alone or with anti-Myc antibody as indicated and imaged by confocal microscopy (e). Scale bars, $10 \mu \mathrm{m}$. (f) Quantification assays of P.M. or C.P. localization of Tube in transfected cells in (e).

mutation of pellino resulted in impaired Drosomycin expression and reduced survival against Gram-positive bacterial infection ${ }^{25}$. In this study, we used a cell-based luciferase-reporter assay system and performed an RNAi knockdown screen. From this screen, we were surprised to find that while knockdown of pellino increased activation of the Drosomycin promoter induced by overexpression of active Toll receptor, overexpression of Pellino suppressed the activity of Toll signalling in a dose-dependent manner in luciferase reporter assays. These findings suggest that Drosophila Pellino has a negative role in the regulation of Toll signalling in S2 cells. We further assessed the in vivo function of Pellino by generating knockdown and overexpression of Pellino transgenes. Notably, tissue-specific knockdown or overexpression of pellino in the fat body supported the findings that Pellino negatively regulates Toll signalling activity in vivo. In addition, alteration of pellino in the fat body influenced $M$. luteus growth in host flies, further emphasizing the biological impact of pellino in negatively regulating the innate immune response. Collectively, our findings strongly support the idea that Drosophila Pellino plays a negative rather than positive role in the Toll signalling pathway.

Drosophila Pellino was initially identified as a Pelle-interacting protein ${ }^{19}$. However, the molecular basis of the action of Drosophila Pellino in Toll signalling remains unknown. In this study, we demonstrated that Pellino not only forms a complex with Pelle but also associates with both Tube and MyD88. Thus, Pelle, Tube and/or MyD88 could be the downstream target(s) of Pellino, given that Pellino functions as a ubiquitin E3 ligase ${ }^{20,21}$. 
a

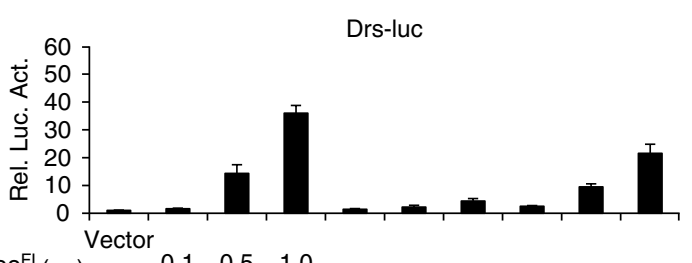

$\begin{array}{llll}\mathrm{MyD}^{2} 8^{\mathrm{FL}}(\mu \mathrm{g}): & 0.1 & 0.5 & 1.0\end{array}$

$\begin{array}{llll}\operatorname{MyD} 8^{\Delta C T E}(\mu \mathrm{g}): & 0.1 & 0.5 & 1.0\end{array}$ $\operatorname{MyD} 88^{\text {CTE-SRC }}(\mu \mathrm{g})$ :

b

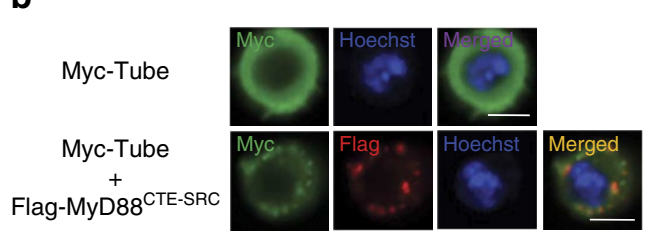

$0.1 \quad 0.5 \quad 1.0$

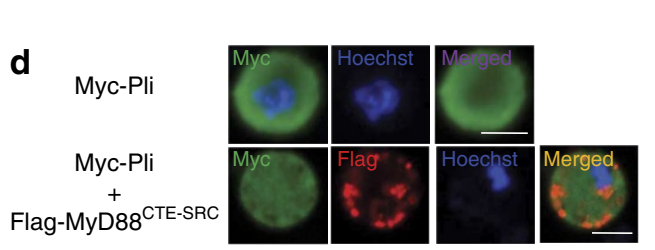

C

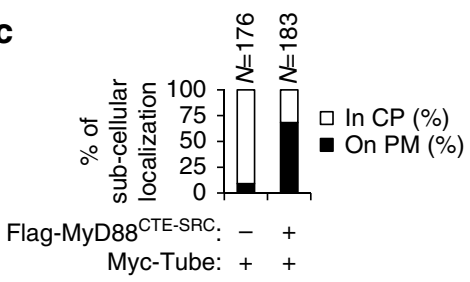

e

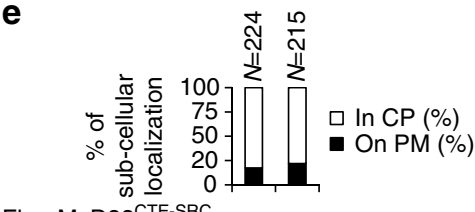

Flag-MyD88 ${ }^{\text {CTE-SRC: }}-+$

g Drs-luc

$$
h^{\text {Myc-Pli: }++} \text { Drs-luc }
$$

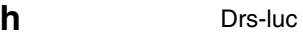

f

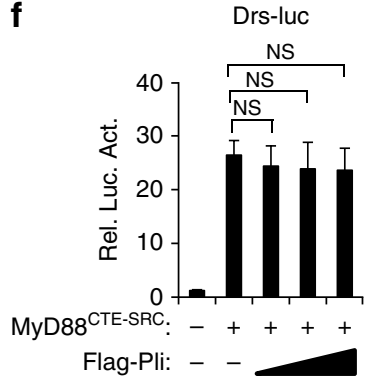

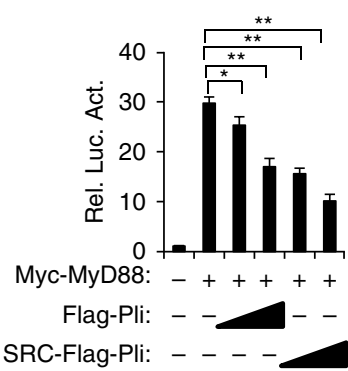

sicherien h

Figure 7 | Cell-surface localization of MyD88 and Pellino balances Toll-mediated immune signalling. (a) S2 cells were transiently transfected with

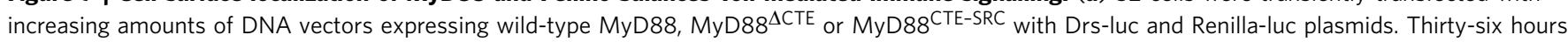
after transfection, luciferase activity was measured and normalized based on Renilla luciferase activity. Results are presented relative to the luciferase activity in control cells. Error bars represent s.d. $(n=3)$. (b,c) S2 cells transfected with Myc-Tube alone or in combination with Flag-tagged MyD88 CTE-SRC were stained with Hoechst and anti-Myc antibody alone or with anti-Flag antibody as indicated and imaged by confocal microscopy (b). Scale bars,

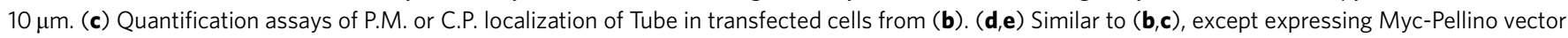
was used. Scale bars, $10 \mu \mathrm{m}$. (f) Luciferase reporter assays showing the effects of increasing expression of Pellino on luciferase activity induced by expression of MyD88 ${ }^{\mathrm{CTE}-S R C}$. S2 cells were transfected with vector or expression constructs as indicated. Thirty-six hours after transfection, cells were extracted for reporter assay. Error bars represent s.d. $(n=3)$. $(\mathbf{g}, \mathbf{h})$ Luciferase reporter assays showing the effects of increasing expression of wild-type Pellino or the SRC-tagged Pellino on luciferase activity induced by expression of wild-type MyD88 (g) or SRC-Pellino on the luciferase activity induced by expression of MyD88 ${ }^{\mathrm{CTE}-\mathrm{SRC}}(\mathbf{h})$. S2 cells were transfected with vector or expression constructs as indicated. Thirty-six hours after transfection, cells were lysed for reporter assay. Error bars represent s.d. $(n=3)$. For data from $\mathbf{f}-\mathbf{h}$, the two-tailed Student's $t$-test was used to analyse statistical significance. ${ }^{\star} P<0.05,{ }^{\star \star} P<0.01$, NS, no significance, versus control groups.

Our biochemical assays showed that alteration of Pellino by knockdown or overexpression markedly changed the ubiquitination pattern and stability status of MyD88, upon pathway activation, indicating MyD88 is the primary target of Pellino in the Toll signalling pathway. A recent study showed MyD88 functions as an adaptor of the Toll receptor and is mainly present at the plasma membrane to activate the downstream events of Toll/MyD88 (ref. 17). Interestingly, we demonstrated that upon bacterium stimulation, high levels of Pellino accumulated onto the plasma membrane, which was dependent on MyD88 activity. Thus, the plasma membrane localization of Pellino might be important for its targeting of MyD88, which then downregulates Toll signalling activity. In support of this, we found that expression of the plasma membrane-tagged Pellino form, SRC-Pellino, strongly enhanced its activity in downregulation of Toll signalling, compared with its wild-type form. Given that MyD88 is associated with Pellino, we have identified a novel feedback mechanism by which MyD88 recruits Pellino onto the plasma membrane, where Pellino targets MyD88 for ubiquitination and degradation.

In mammals, it has been shown that the function of Pellino proteins is regulated by the IRAK-1, the homologue of Pelle, through phosphorylation in a site-specific manner. Interestingly, we found that some of these sites are also present in Drosophila Pellino. By generating a series of mutant forms of Pellino, in which the $\mathrm{S}$ or $\mathrm{T}$ was individually or in combination mutated to 
A, we found that these mutations did not affect the function of Pellino, as indicated by luciferase reporter assays (Supplementary Fig. 9a), as well as the interaction of Pellino with MyD88 in co-immunoprecipitation assays (Supplementary Fig. 9b). Moreover, Pellino carrying these mutations could accumulate at plasma membrane upon Toll signalling activation (Supplementary Fig. 9c,d). Collectively, the conserved sites are not required for E3 ligase activity of Drosophila Pellino in the regulation of MyD88.

Pathways involving signalling transduction are most often initiated by transmembrane receptors, which typically possess separate domains for engaging extracellular ligands and intracellular adaptor proteins $s^{15}$. In the case of Toll signalling pathway, the plasma membrane localization of MyD88 is necessary for Toll signalling transduction by recruiting the downstream adaptor protein Tube onto the plasma membrane ${ }^{17,18}$. Drosophila MyD88 contains a phosphoinositide-binding domain (named CTE) at its $\mathrm{C}$ terminus, which is proposed to function as a 'SORT' adaptor for MyD88 and Tube cell-surface localization, and subsequently for downstream signalling transduction of the Toll pathway ${ }^{17}$. The CTE domain of MyD88 is the functional homologue of TIRAP in mammals ${ }^{17,41,42}$, and thus positively regulates Toll signalling. In this study, in addition to its positive role in Toll signalling, MyD88 CTE was sufficient to interact with and promoted Pellino cell-surface localization. The plasma membrane localization of Pellino allows the negative control of MyD88 ubiquitination and degradation in a feedback regulatory manner. Thus, the dual roles of the MyD88 CTE domain are essential for both the activation and negative maintenance of homeostasis of Toll signalling.

The endocytosis is a dynamic cellular process controlling various signalling pathways in either a positive or a negative manner. Recently, several studies have shown that the endocytic pathway is involved in regulating the Toll signalling transduction in Drosophila ${ }^{43,44}$. It has been shown that the endosomal proteins, such as Mop and Hrs, colocalize with the Toll receptor in endosomes and function genetically upstream of MyD88 and Pelle; thus, endocytosis is required for the Toll signalling activation. Internalization and trafficking of the nascent receptor to some particular endosomes has been proposed to extend signalling activity, as in the cases of Notch and JAK/STAT signalling pathways in Drosophila ${ }^{45,46}$. In support of this, we found that expression of the hyper-activated form of MyD88, MyD88CTE-SRC, not only showed a plasma membrane localization but also exhibited a particulate distribution pattern in cytosolic region. Notably, we observed that addition of pretreated M. luteus into S2 cells could promote the Pellino plasma membrane localization (Fig. 5a) and activate Toll signalling, albeit at a relative lower level, when compared with the control cells stimulated by activated Toll receptor. Knockdown of Croquemort ( $\mathrm{crq}$ ), a component of phagocytosis pathway ${ }^{47}$, significantly suppressed Pellino plasma membrane localization and upregulation of metchnikowin, defensin and drosomycin induced by M. luteus in S2 cells (Supplementary Fig. 10a-h). These findings together emphasize the significant roles of some specific type of endocytic (or phagocytic) vesiclemediated Toll signalling pathway. It would be interesting to test how the endocytic (or phagocytic) pathway contributes to the regulation of Drosophila innate immunity in the future studies.

\section{Methods}

Drosophila strains. Fly stocks were maintained under standard culture conditions. The $w^{1118}$ strain was used as a control and the host for P-element-mediated transformation ${ }^{48}$. The following strains were used in this study: (1) $\mathrm{P}\{U A S p$ pellino , in which the full-length pellino was placed under the control of the UASp promoter ${ }^{49}$; (2) Pellino knockdown transgenes, $\mathrm{P}\{U A S p$-ArtmiR-pellino $\}$ that express artificial microRNAs targeting pellino mRNA were generated by the following method. Briefly, the two pairs of designed primers were denatured and annealed to form short dsDNA, which were then digested by NheI and EcoRI enzymes to insert into UASp KN and BX vectors, respectively. The two inserted UASp vectors were then digested with BamHI and XbaI enzymes, producing a short DNA and a broken vector. The final UASp-ArtmiR-pellino vector was obtained when the short DNA from digested UASp KN vector was inserted into the broken UASp BX vector; (3) the Cg-gal4, a fat-body-specific gal4 driver, was a kind gift from Dr Sheng Li (Shanghai Institutes for Biological Science, CAS); (4) P $\{t u b P$ gal4\} was from the Bloomington Stock Center (BL5138). (5) The ubiquitous Gal4Gal80ts driver system was used to express the target genes specifically at the adult stage. Crosses were performed and the resulting progeny were initially raised the permissive temperature $\left(18^{\circ} \mathrm{C}\right)$ for the Gal80ts inhibitor to repress the Gal4 activity. Once adult eclosion, the newly progeny were then shifted to $29^{\circ} \mathrm{C}$ to allow activation of Gal4 at the adult stage.

Primers used for transgene vector construction are shown in Supplementary Table 1.

Immunoprecipitation and western blot assay. S2 cells were cultured in insect medium (Hyclone) at $27^{\circ} \mathrm{C}$. DNA transfection was performed using Lipofectamine 2000 (Invitrogen) or using the standard calcium phosphate transfection method. For immunoprecipitation assays, cells were lysed in lysis buffer $(150 \mathrm{mM} \mathrm{NaCl}$, $50 \mathrm{mM}$ Tris- $\mathrm{HCl}, \mathrm{pH} 7.4,10 \%$ glycerol, $0.5 \%$ Triton X-100, $10 \mu \mathrm{g} \mathrm{ml}^{-1}$ aprotinin, $10 \mu \mathrm{g} \mathrm{ml}^{-1}$ leupeptin and $1 \mathrm{mM}$ phenylmethylsulfonyl fluoride). Anti-Flag M2 affinity gel (Sigma) or anti-Myc affinity gel (Abmart) were used for indicated immunoprecipitation experiments. The immune complexes were washed three times with lysis buffer containing $150 \mathrm{mM} \mathrm{NaCl}$ and subjected to immunoblot analysis with the indicated antibodies. Immunoblotting was performed according to standard procedures. The following antibodies were used for western blotting: rabbit anti-Myc (1:3,000, MBL), rabbit anti-HA (1:3,000, MBL), rabbit or mouse anti-Flag (1:2,000, Sigma) and rabbit anti-Actin (1:2,000, Sigma). The antibody against Pellino was generated by immunizing rabbits with the recombinant proteins $\mathrm{His}_{6}$-Pellino (1-120) produced in E. coli. The antibodies against MyD88, Drosomycin and Defensin were generated by immunizing mice with the E. coliproduced recombinant proteins $\mathrm{His}_{6}$-MyD88 (63-163), Drosomycin (full-length) and Defensin (full-length) respectively. Original western blots can be found in Supplementary Fig. 11 .

In vivo ubiquitination assay. In vivo ubiquitination assays were performed by the following method. Briefly, S2 cells were transfected with the indicated DNA constructs. At $48 \mathrm{~h}$ post-transfection, cells were treated with MG132 (final concentration $50 \mu \mathrm{M}$ ) and/or $\mathrm{NH}_{4} \mathrm{Cl}$ (final concentration $50 \mathrm{mM}$ ) for $6 \mathrm{~h}$. Cells were lysed in lysis buffer $(150 \mathrm{mM} \mathrm{NaCl}, 50 \mathrm{mM}$ Tris- $\mathrm{HCl}, \mathrm{pH} 7.4,10 \%$ glycerol $1 \%$ Triton X-100, $0.1 \%$ SDS and $10 \mathrm{mM} \mathrm{NEM}$ ). After pull-down with anti-Myc affinity beads for $4 \mathrm{~h}$, the beads were extensively washed three times with lysis buffer containing $0.1 \%$ SDS and $500 \mathrm{mM} \mathrm{NaCl}$ for a total of $1 \mathrm{~h}$. Samples were then subjected to western blot analysis.

Toll and IMD signalling reporter assay in S2 cells. Toll and IMD signalling reporter assays in S2 cells were performed using the drosomycin-luciferase or attacin-luciferase constructs, in which the luciferase coding sequence was placed under the drosomycin or attacin promoter. For normalizing the efficiency of the transfection, the actinP-Renilla construct was used. Firefly luciferase and Renilla luciferase were performed by the following method. In brief, the S2 cells were lysed with $50 \mu \mathrm{l}$ of Passive lysis buffer (Promega) for luciferase assays using Luciferase assay reagent (Promega), according to the manufacturer's instructions.

Toll signalling induced by pre-boiled $\boldsymbol{M}$. luteus. $M$. luteus was cultured overnight in $30^{\circ} \mathrm{C}$ and harvested when the $\mathrm{OD}_{600}$ of the culture material reached 1.00 . The bacteria collection was then diluted 10 times with PBS buffer and boiled for 20 minutes. S2 cells were treated with this pre-boiled bacterial mixture for $12 \mathrm{~h}$ and then harvested for quantitative RT-PCR or immunostaining assays.

RNAi knockdown assays in S2 cells. All dsRNAs were synthesized according to the standard protocol. S2 cells were collected and diluted into fresh medium at a density of $1.0 \times 10^{6}$ cells per ml, and then were treated with dsRNA immediately. Primers for dsRNA synthesis are shown in Supplementary Table 2.

Quantitative RT-PCR. Total RNA was isolated with Trizol Reagent (Invitrogen) according to the manufacturer's instructions and CDNA was synthesized using SuperScript III First-Strand cDNA Synthesis kit (Invitrogen). Quantitative RT-PCR was performed using SYBR Premix Ex Taq (Takara) in triplicate on a Bio-Rad iCycler iQ5 PCR Thermal Cycler. Template concentrations were normalized to endogenous reference rp49. Primers for quantitative RT-PCR are shown in Supplementary Table 3. 
Strains of bacteria. All the bacterium strains used in this work were obtained from the China General Microbiological Culture Collection Center (CGMCC). The CGMCC numbers for Micrococcus luteus, Enterococcus faecalis and Aspergillus fumigatus are $1.2299,1.2135$ and 3.08027 , respectively.

Survival and bacterial CFU assays in flies. Bacterial challenge was performed by the following method. Briefly, a thin needle dipped into a concentrated overnight culture of M. luteus was used to prick 6-day-old adult flies for bacterial infection. After infection, flies were immediately transferred to fresh vials every day. Survival experiments were performed in the same conditions for each tested genotype, and 30 flies per group were tested for each genotype. The numbers of surviving flies were counted every day. Flies that died within $3 \mathrm{~h}(<5 \%$ of the total $)$ after challenge were not considered in the analysis.

For CFU counting assays, 10 infected flies were harvested at the indicated times after infection and crushed in PBS buffer. The mixture was diluted serially, and $100 \mu \mathrm{l}$ of each dilution was spread onto LB agar plates. Yellow bacterial clones were counted after overnight growth in a $30^{\circ} \mathrm{C}$ culture hood.

Cell immunofluorescence. For cell immunostaining experiments, cells were transferred onto a poly-lysine-treated cover glass $12 \mathrm{~h}$ after transfection. Twentyfour hours later, cells were fixed with $4 \%$ formaldehyde in PBS containing $0.1 \%$ Tween-20 for $15 \mathrm{~min}$, permeabilized and blocked with $0.3 \%$ Tween-20 in PBS containing 5\% BSA for $15 \mathrm{~min}$ at room temperature. Cells were then incubated with primary antibody and secondary antibody. Imaging of the cells was performed using a Zeiss LSM 710 META laser scanning confocal system. The following antibodies were used in this study: mouse anti-Flag (1:2,000, Sigma) and rabbit anti-Myc $(1: 2,000, \mathrm{MBL})$. The following secondary antibodies were used at a 1:3,000 dilution: goat anti-mouse Alexa555 and goat anti-rabbit Alexa488.

Two-tailed Student's t-test. The data of reporter and quantitative RT-PCR assays in this study were collected from three biological replicates. All statistical analyses are shown as means \pm s.d. Significant differences between values under different experimental conditions were determined by two-tailed Student's $t$-test. For all tests, a $P$-value $<0.05$ was considered statistically significant. ${ }^{\star} P<0.05,{ }^{\star \star} P<0.01$, ${ }^{* * *} P<0.001$, NS, no significance, versus control groups.

Log-rank test. The data of survival and protein stability assays were collected from three biological replicates. All statistical analyses are shown as means \pm s.d. Significant differences were analysed by Log-rank test using the PASW Statistics 18 software. For all tests, a $P$-value $<0.05$ was considered statistically significant.

\section{Repeated measures analysis of variance for bacterial loading. The data of} bacterial loading assays were collected from six biological replicates. The rANOVA, a commonly used statistical approach for repeated-measure designs, was used to analyse the variance of the bacterial loading assays. With such designs, the repeated-measure factor (the qualitative independent variable) is the with-subjects factor, while the dependent quantitative variable on which each participant is measured is the dependent variable. All statistical analyses results are shown as $x y$ scatter diagrams. The variance analyses of these repeated measures were finished using the PASW Statistics 18 software. The $P$-value showed the results of the between-subjects effect tests.

Flybase numbers for genes used in this study. The mRNA sequence data for genes of Drosophila described in this study can be found in the Flybase under the following accession numbers: Toll (NP 524518), Myd88 (NP 610479), Pelle (NP_476971), Tube (NP_001189164), Pellino (NP_524466) and PGRP-LCa (NP_729468).

\section{References}

1. Akira, S., Uematsu, S. \& Takeuchi, O. Pathogen recognition and innate immunity. Cell 124, 783-801 (2006).

2. Alarco, A. M. et al. Immune-deficient Drosophila melanogaster: a model for the innate immune response to human fungal pathogens. J. Immunol. 172, 5622-5628 (2004).

3. Hultmark, D. Drosophila immunity: paths and patterns. Curr. Opin. Immunol. 15, 12-19 (2003).

4. Hoffmann, J. A. The immune response of Drosophila. Nature 426, 33-38 (2003).

5. Tanji, T., Hu, X., Weber, A. N. \& Ip, Y. T. Toll and IMD pathways synergistically activate an innate immune response in Drosophila melanogaster. Mol. Cell Biol. 27, 4578-4588 (2007).

6. Tanji, T. \& Ip, Y. T. Regulators of the Toll and Imd pathways in the Drosophila innate immune response. Trends Immunol. 26, 193-198 (2005).

7. Hedengren, M. et al. Relish, a central factor in the control of humoral but not cellular immunity in Drosophila. Mol. Cell 4, 827-837 (1999).
8. Han, Z. S. \& Ip, Y. T. Interaction and specificity of Rel-related proteins in regulating Drosophila immunity gene expression. J. Biol. Chem. 274, 21355-21361 (1999).

9. Lemaitre, B., Nicolas, E., Michaut, L., Reichhart, J. M. \& Hoffmann, J. A. The dorsoventral regulatory gene cassette spatzle/Toll/cactus controls the potent antifungal response in Drosophila adults. Cell 86, 973-983 (1996).

10. Meng, X., Khanuja, B. S. \& Ip, Y. T. Toll receptor-mediated Drosophila immune response requires Dif, an NF-kappaB factor. Genes Dev. 13, 792-797 (1999).

11. Ligoxygakis, P., Pelte, N., Hoffmann, J. A. \& Reichhart, J. M. Activation of Drosophila Toll during fungal infection by a blood serine protease. Science 297, 114-116 (2002).

12. Horng, T. \& Medzhitov, R. Drosophila MyD88 is an adapter in the Toll signaling pathway. Proc. Natl. Acad. Sci. USA 98, 12654-12658 (2001).

13. Tauszig-Delamasure, S., Bilak, H., Capovilla, M., Hoffmann, J. A. \& Imler, J. L. Drosophila MyD88 is required for the response to fungal and Gram-positive bacterial infections. Nat. Immunol. 3, 91-97 (2002).

14. Rutschmann, S. et al. The Rel protein DIF mediates the antifungal but not the antibacterial host defense in Drosophila. Immunity 12, 569-580 (2000).

15. Haugh, J. M. A unified model for signal transduction reactions in cellular membranes. Biophys. J. 82, 591-604 (2002).

16. Kholodenko, B. N., Hoek, J. B. \& Westerhoff, H. V. Why cytoplasmic signalling proteins should be recruited to cell membranes. Trends Cell Biol. 10, 173-178 (2000).

17. Marek, L. R. \& Kagan, J. C. Phosphoinositide binding by the Toll adaptor $\mathrm{dMyD} 88$ controls antibacterial responses in Drosophila. Immunity 36, 612-622 (2012).

18. Sun, H., Towb, P., Chiem, D. N., Foster, B. A. \& Wasserman, S. A. Regulated assembly of the Toll signaling complex drives Drosophila dorsoventral patterning. EMBO J. 23, 100-110 (2004).

19. Grosshans, J., Schnorrer, F. \& Nusslein-Volhard, C. Oligomerisation of Tube and Pelle leads to nuclear localisation of dorsal. Mech. Dev. 81, 127-138 (1999).

20. Moynagh, P. N. The Pellino family: IRAK E3 ligases with emerging roles in innate immune signalling. Trends Immunol. 30, 33-42 (2009).

21. Jin, W., Chang, M. \& Sun, S. C. Peli: a family of signal-responsive E3 ubiquitin ligases mediating TLR signaling and T-cell tolerance. Cell Mol. Immunol. 9, 113-122 (2012).

22. Kim, T. W. et al. Pellino 2 is critical for Toll-like receptor/interleukin-1 receptor (TLR/IL-1R)-mediated post-transcriptional control. J. Biol. Chem. 287, 25686-25695 (2012).

23. Xiao, H. et al. Pellino $3 \mathrm{~b}$ negatively regulates interleukin-1-induced TAK1dependent NF kappaB activation. J. Biol. Chem. 283, 14654-14664 (2008).

24. Jiang, Z. et al. Pellino 1 is required for interleukin-1 (IL-1)-mediated signaling through its interaction with the IL-1 receptor-associated kinase 4 (IRAK4)IRAK-tumour necrosis factor receptor-associated factor 6 (TRAF6) complex. J. Biol. Chem. 278, 10952-10956 (2003).

25. Haghayeghi, A., Sarac, A., Czerniecki, S., Grosshans, J. \& Schock, F. Pellino enhances innate immunity in Drosophila. Mech. Dev. 127, 301-307 (2010).

26. Ferrandon, D. et al. A drosomycin-GFP reporter transgene reveals a local immune response in Drosophila that is not dependent on the Toll pathway. ЕМВО J. 17, 1217-1227 (1998).

27. Tauszig, S., Jouanguy, E., Hoffmann, J. A. \& Imler, J. L. Toll-related receptors and the control of antimicrobial peptide expression in Drosophila. Proc. Natl Acad. Sci. USA 97, 10520-10525 (2000).

28. van Wijk, S. J. et al. A comprehensive framework of E2-RING E3 interactions of the human ubiquitin-proteasome system. Mol. Syst. Biol. 5, 295 (2009).

29. Deshaies, R. J. \& Joazeiro, C. A. RING domain E3 ubiquitin ligases. Annu. Rev. Biochem. 78, 399-434 (2009).

30. Gottar, M. et al. The Drosophila immune response against Gram-negative bacteria is mediated by a peptidoglycan recognition protein. Nature $\mathbf{4 1 6}$, 640-644 (2002).

31. Schmidt, R. L. et al. Cleavage of PGRP-LC receptor in the Drosophila IMD pathway in response to live bacterial infection in S2 cells. Self Nonself 2, 125-141 (2011).

32. Rus, F. et al. Ecdysone triggered PGRP-LC expression controls Drosophila innate immunity. EMBO J. 32, 1626-1638 (2013).

33. Wang, H., Mu, Y. \& Chen, D. Effective gene silencing in Drosophila ovarian germline by artificial microRNAs. Cell Res. 21, 700-703 (2011).

34. Deng, L. et al. Activation of the IkappaB kinase complex by TRAF6 requires a dimeric ubiquitin-conjugating enzyme complex and a unique polyubiquitin chain. Cell 103, 351-361 (2000).

35. Huang, A. M., Rusch, J. \& Levine, M. An anteroposterior Dorsal gradient in the Drosophila embryo. Genes Dev. 11, 1963-1973 (1997).

36. Braun, A., Hoffmann, J. A. \& Meister, M. Analysis of the Drosophila host defence in domino mutant larvae, which are devoid of hemocytes. Proc. Natl Acad. Sci. USA 95, 14337-14342 (1998).

37. Xia, L. et al. The Fused/Smurf complex controls the fate of Drosophila germline stem cells by generating a gradient BMP response. Cell 143, 978-990 (2010). 
38. Glickman, M. H. \& Ciechanover, A. The ubiquitin-proteasome proteolytic pathway: destruction for the sake of construction. Physiol. Rev. 82, 373-428 (2002).

39. Spencer, E., Jiang, J. \& Chen, Z. J. Signal-induced ubiquitination of IkappaBalpha by the F-box protein Slimb/beta-TrCP. Genes Dev. 13, 284-294 (1999).

40. Shen, B., Liu, H., Skolnik, E. Y. \& Manley, J. L. Physical and functional interactions between Drosophila TRAF2 and Pelle kinase contribute to Dorsal activation. Proc. Natl Acad. Sci. USA 98, 8596-8601 (2001).

41. Horng, T., Barton, G. M., Flavell, R. A. \& Medzhitov, R. The adaptor molecule TIRAP provides signalling specificity for Toll-like receptors. Nature 420, 329-333 (2002).

42. Yamamoto, M. et al. Essential role for TIRAP in activation of the signalling cascade shared by TLR2 and TLR4. Nature 420, 324-329 (2002).

43. Huang, H. R., Chen, Z. J., Kunes, S., Chang, G. D. \& Maniatis, T. Endocytic pathway is required for Drosophila Toll innate immune signaling. Proc. Nat Acad. Sci. USA 107, 8322-8327 (2010).

44. Lund, V. K., DeLotto, Y. \& DeLotto, R. Endocytosis is required for Toll signaling and shaping of the Dorsal/NF-kappaB morphogen gradient during Drosophila embryogenesis. Proc. Natl Acad. Sci. USA 107, 18028-18033 (2010).

45. Devergne, O., Ghiglione, C. \& Noselli, S. The endocytic control of JAK/STAT signalling in Drosophila. J. Cell Sci. 120, 3457-3464 (2007).

46. Vaccari, T., Lu, H., Kanwar, R., Fortini, M. E. \& Bilder, D. Endosomal entry regulates Notch receptor activation in Drosophila melanogaster. J. Cell Biol. 180, 755-762 (2008).

47. Franc, N. C., Heitzler, P., Ezekowitz, R. A. \& White, K. Requirement for croquemort in phagocytosis of apoptotic cells in Drosophila. Science 284, 1991-1994 (1999).

48. Rubin, G. M. \& Spradling, A. C. Genetic transformation of Drosophila with transposable element vectors. Science 218, 348-353 (1982).

49. Rorth, P. Gal4 in the Drosophila female germline. Mech. Dev. 78, 113-118 (1998).

\section{Acknowledgements}

We would like to thank Dr Sheng Li (Shanghai Institutes for Biological Science, CAS) for generously providing Cg-gal4 fly strain. This work was supported by grants from the National Basic Research Program of China (2010CB945300 and 2013CB945000), National Natural Science Foundation of China (31370882 and 31130036).

\section{Author contributions}

S.J., D.C. and Q.S. conceived and designed the experiments. S.J., M.S., L.L. and L.S. performed the experiments. S.J., M.S., D.C. and Q.S. analysed the data. X.Z., S.J performed all statistical analysis. S.J., D.C. and Q.S. wrote the manuscript.

\section{Additional information}

Supplementary Information accompanies this paper at http://www.nature.com/ naturecommunications

Competing financial interests: The authors declare no competing financial interests.

Reprints and permission information is available online at http://npg.nature.com/ reprintsandpermissions/

How to cite this article: Ji, S. et al. Cell-surface localization of Pellino antagonizes Toll-mediated innate immune signalling by controlling MyD88 turnover in Drosophila. Nat. Commun. 5:3458 doi: 10.1038/ncomms4458 (2014).

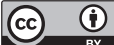

This work is licensed under a Creative Commons Attribution 3.0 Unported License. The images or other third party material in this article are included in the article's Creative Commons license, unless indicated otherwise in the credit line; if the material is not included under the Creative Commons license, users will need to obtain permission from the license holder to reproduce the material. To view a copy of this license, visit http://creativecommons.org/licenses/by/3.0/ 